ARTICLE

\title{
Trans- and cis-acting effects of Firre on epigenetic features of the inactive $X$ chromosome
}

He Fang ${ }^{1,10}$, Giancarlo Bonora (10 2,10, Jordan P. Lewandowski ${ }^{3}$, Jitendra Thakur ${ }^{4}$, Galina N. Filippova ${ }^{1}$,

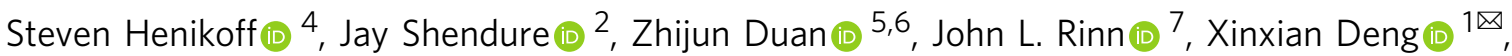
William S. Noble (id ${ }^{2,8 \times} \&$ Christine M. Disteche (id ${ }^{1,9 凶}$

Firre encodes a IncRNA involved in nuclear organization. Here, we show that Firre RNA expressed from the active $X$ chromosome maintains histone H3K27me3 enrichment on the inactive $X$ chromosome $\left(X_{i}\right)$ in somatic cells. This trans-acting effect involves SUZ12, reflecting interactions between Firre RNA and components of the Polycomb repressive complexes. Without Firre RNA, H3K27me3 decreases on the $\mathrm{Xi}$ and the Xi-perinucleolar location is disrupted, possibly due to decreased CTCF binding on the Xi. We also observe widespread gene dysregulation, but not on the $\mathrm{Xi}$. These effects are measurably rescued by ectopic expression of mouse or human Firre/FIRRE transgenes, supporting conserved transacting roles. We also find that the compact 3D structure of the Xi partly depends on the Firre locus and its RNA. In common lymphoid progenitors and T-cells Firre exerts a cis-acting effect on maintenance of $\mathrm{H} 3 \mathrm{~K} 27 \mathrm{me} 3$ in a $26 \mathrm{Mb}$ region around the locus, demonstrating cell typespecific trans- and cis-acting roles of this IncRNA.

\footnotetext{
${ }^{1}$ Department of Laboratory Medicine and Pathology, University of Washington, Seattle, WA, USA. ${ }^{2}$ Department of Genome Sciences, University of Washington, Seattle, WA, USA. ${ }^{3}$ Department of Stem Cell and Regenerative Biology, Harvard University, Boston, MA, USA. ${ }^{4}$ Fred Hutchinson Cancer Research Center, Seattle, WA, USA. ${ }^{5}$ Institute for Stem Cell and Regenerative Medicine, University of Washington, Seattle, WA, USA. ${ }^{6}$ Division of Hematology, Department of Medicine, University of Washington, Seattle, WA, USA. 7 Department of Biochemistry, University of Colorado at Boulder, Boulder, CO, USA. ${ }^{8}$ Paul G. Allen School of Computer Science and Engineering, University of Washington, Seattle, WA, USA. ${ }^{9}$ Department of Medicine,

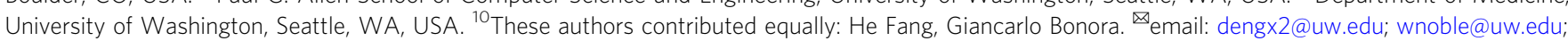
cdistech@uw.edu
} 
X chromosome inactivation (XCI) is initiated by the long noncoding RNA (lncRNA) Xist, which becomes highly expressed on one allele, and coats the future inactive $\mathrm{X}$ chromosome $(\mathrm{Xi})$ in cis $^{1-5}$. Specific proteins that include components of the Polycomb repressive complexes PRC1 and PRC2 are recruited by Xist RNA to mediate serial layers of epigenetic modifications, resulting in gene silencing and heterochromatin formation ${ }^{2,6,7}$. Epigenetic hallmarks of the Xi include multiple repressive histone modifications such as ubiquitination of histone $\mathrm{H} 2 \mathrm{~A}$ at lysine 119 (H2AK119ubi), tri-methylation of histone H3 at lysine 27 (H3K27me3), and enrichment in the histone variant macroH $2 \mathrm{~A}^{8}{ }^{8}$. Additional layers of control ensure stability of the silent state of the $\mathrm{Xi}$, including DNA methylation of promotercontaining $\mathrm{CpG}$ islands, a shift to late replication, and spatial reorganization of the $\mathrm{Xi}$ within the nucleus ${ }^{9,10}$.

The Xi appears as the heteropycnotic Barr body usually located close to either the nuclear lamina or the periphery of the nucleolus ${ }^{1-15}$. These two locations are preferred sites of heterochromatin, not only for the Xi but also for other repressed regions of the genome, suggesting that their proximity helps maintain silent chromatin ${ }^{11,16}$. In particular, the perinucleolar space has a primary function in replication and maintenance of repressive chromatin state $^{17,18}$. The factors and mechanisms that facilitate association of heterochromatic regions including the $\mathrm{Xi}$ to specific nuclear compartments such as the lamina or the nucleolus remain elusive. Xist RNA interaction with the lamin B receptor (LBR) has been proposed as a critical factor that recruits the $\mathrm{Xi}$ to the lamina and facilitates silencing ${ }^{19}$. Our previous studies suggest that perinucleolar positioning of the Xi may be facilitated by the lncRNA Firre ${ }^{20}$.

The Firre locus comprises conserved tandem repeats that bind CTCF specifically on the Xi but not on the Xa (active X chromosome $)^{20-22}$. Despite sequence divergence between species, the conserved nature of the repeat locus suggests important roles in mammals ${ }^{21}$. Firre RNA is usually confined to the nucleus where it interacts with the nuclear matrix protein hnRNPU 23,24 . Multiple transcript isoforms including circular RNAs, further complicate an understanding of the roles of Firre in different cell types ${ }^{25}$. On the $\mathrm{Xi}$ the Firre locus contacts the Dxz4 locus that also binds CTCF only on the $\mathrm{Xi}^{26-28}$. Dxz4 is necessary for the formation of the bipartite structure of the $\mathrm{Xi}^{27,29-31}$. The Firre locus also interacts with several autosomal regions, consistent with a widespread role in nuclear architecture ${ }^{23,32}$. A Firre knockout (KO) mouse model is viable, but results in cell-type-specific defects in hematopoiesis that impact common lymphoid progenitors (CLPs) ${ }^{32,33}$. Importantly, these defects are rescued by ectopic expression of Firre from an autosomal location, thus defining a trans-acting role for Firre ${ }^{32,33}$. KO mice show organspecific dysregulation of autosomal genes, consistent with physiological defects in distinct phases of hematopoiesis ${ }^{32}$.

Here, we investigate the role of Firre in maintenance of heterochromatin, gene expression, and 3D structure of the $\mathrm{Xi}$ by engineering allele-specific deletions of the Firre locus and by Firre knockdown (KD) in mouse cell lines and tissues. Depletion of Firre RNA reveals important roles in $\mathrm{H} 3 \mathrm{~K} 27$ me3 enrichment on the $\mathrm{Xi}$ and in location of the $\mathrm{Xi}$ within the nucleus as shown by immunostaining, ChIP-seq, and CUT\&RUN. Gene expression is disrupted, as is the $3 \mathrm{D}$ structure of the $\mathrm{Xi}$ as shown by RNA-seq, ATAC-seq, and Hi-C. Our results are supported by rescue experiments using cDNA transgenes. We demonstrate both trans- and cis-acting roles of Firre RNA and its locus, with evidence of cell-type-specific effects in cell lines and in vivo.

\section{Results}

Firre and CrossFirre are transcribed from the Xa. Allele-specific CRISPR/Cas9 editing of the Firre region was done in Patski cells, in which skewed XCI and species-specific SNPs allowed us to design guides to target the $\mathrm{Xi}$ from BL6 or the Xa from Mus spretus (spretus) (Supplementary Data 1$)$. We isolated single-cell clones with either a $\sim 160 \mathrm{~kb}$ deletion of Firre on the Xa ( $\Delta$ Fir$\left.r e^{\mathrm{Xa}}\right)$, a $\sim 160 \mathrm{~kb}$ deletion of Firre on the $\mathrm{Xi}\left(\Delta\right.$ Firre $\left.^{\mathrm{Xi}}\right)$, or a $\sim 160 \mathrm{~kb}$ inversion of Firre on the Xi (InvFirre ${ }^{\mathrm{Xi}}$ ) (Fig. 1a). Deletion of the Firre locus on the Xa resulted in undetectable Firre expression by RT-PCR, while deletion on the Xi caused no change (Fig. 1a, b, Supplementary Data 2). Allele-specific RNAseq analysis confirmed the absence of Firre transcripts from either $\mathrm{Xa}$ or $\mathrm{Xi}$ in $\Delta$ Firre $^{\mathrm{Xa}}$, while control loci (Dxz4, Xist) showed no change (Supplementary Data 3).

Our $\Delta$ Firre $^{\mathrm{Xa}}$ deletion includes the antisense transcript CrossFirre that partially overlaps Firre (Fig. 1a; Supplementary Fig. $1 \mathrm{a})^{34}$. Note that, in contrast to $\Delta$ Firre $^{\mathrm{Xa}}$, previously constructed Firre deletions in mouse ES cells and in a KO mouse do not include all of CrossFirre ${ }^{32,35}$. To test CrossFirre expression in edited Patski cells, we processed strand-specific RT-PCR using primers (F1/R1) that flank a $231 \mathrm{bp}$ region in the middle of CrossFirre with no Firre overlap, and primers (F2/R2) that flank a 203 bp region overlapping the 3' end of Firre (Fig. 1a). For the non-overlapped region, forward CrossFirre transcripts containing only SNPs from the Xa (spretus), were present in WT (wild-type), $\Delta$ Firre $^{\mathrm{Xi}}$, and InvFirre ${ }^{\mathrm{Xi}}$, but absent in $\Delta$ Firre $^{\mathrm{Xa}}$ (Fig. 1c, d). For the overlapped region, transcripts from both directions (CrossFirre and Firre), again containing only SNPs from the Xa (spretus), were present in WT, $\Delta$ Firre ${ }^{\mathrm{Xi}}$, and InvFirre ${ }^{\mathrm{Xi}}$, but absent in $\Delta$ Firre $^{\mathrm{Xa}}$ (Fig. 1d). We found no evidence of miRNAs in the Firre/CrossFirre region using miRNA-seq in WT or $\Delta$ Firre ${ }^{\mathrm{Xa}}$, suggesting that the loci function independently of the small RNA pathway (Supplementary Data 4).

We conclude that Firre and CrossFirre are both transcribed from the $\mathrm{Xa}$ in Patski cells. Note that Firre was originally identified as a gene that escapes XCI in human and mouse $\mathrm{e}^{20,23,28}$ However, our current results and those reported in Firre KO mouse ES cells and in KO mice clearly show that Firre is predominantly expressed from the Xa in fibroblasts ${ }^{32,35}$.

Firre acts in trans to maintain PRC2 and H3K27me3 on the Xi. To determine whether any of the allelic alterations constructed, $\Delta$ Firre $^{\mathrm{Xa}}, \Delta$ Firre $^{\mathrm{Xi}}$, and InvFirre ${ }^{\mathrm{Xi}}$, influences epigenetic marks on the $\mathrm{Xi}$, immunostainings for H3K27me3, H2AK119ubi, and macroH2A.1 were done in combination with Xist RNA-FISH to locate the Xi. The majority of nuclei $(95 \pm 3 \%)$ had one Xist cloud in all edited cell lines, indicating no disruption of Xist RNA coating (Fig. 2a). A strong H3K27me3 immunostaining cluster was observed on the $\mathrm{Xi}$ in $83 \pm 2 \%$ of WT nuclei as expected. In contrast, only $9 \pm 2 \%$ of nuclei with a $\mathrm{H} 3 \mathrm{~K} 27 \mathrm{me} 3$ cluster were observed in $\Delta$ Firre $^{\mathrm{Xa}}$, with most nuclei appearing uniformly mottled throughout (Fig. 2b; Table 1). There was no evidence of a complete loss of $\mathrm{H} 3 \mathrm{~K} 27 \mathrm{me} 3$ over the $\mathrm{Xi}$, which would have appeared as a "hole" with complete absence of immunostaining. H3K27me3 level throughout $\Delta$ Firre ${ }^{\mathrm{Xa}}$ and WT nuclei was measured in regions outside the $\mathrm{Xi}$ using ImageJ to quantify fluorescence intensity by normalization to DNA or to histone panH $4^{36}$. No significant difference was detected in regions outside the Xi between $\Delta$ Firre $^{\mathrm{Xa}}$ and WT, but subtle or local changes undetectable by immunostaining cannot be excluded (Supplementary Fig. 2a, b). $\Delta$ Firre $^{\mathrm{Xi}}$ and InvFirre ${ }^{\mathrm{Xi}}$ nuclei retained a strong $\mathrm{H} 3 \mathrm{~K} 27 \mathrm{me} 3$ cluster on the $\mathrm{Xi}$, consistent with retention of Firre RNA in these cells (Fig. 2b). Two other histone modifications known to be associated with XCI, H2AK119ubi and macroH2A.1, showed no changes in $\Delta$ Firre ${ }^{\mathrm{Xa}}$ nuclei (Fig. 2c).

Next, allele-specific profiles of $\mathrm{H} 3 \mathrm{~K} 27 \mathrm{me} 3$ were generated by ChIP-seq, which demonstrated a chromosome-wide decrease on 
a

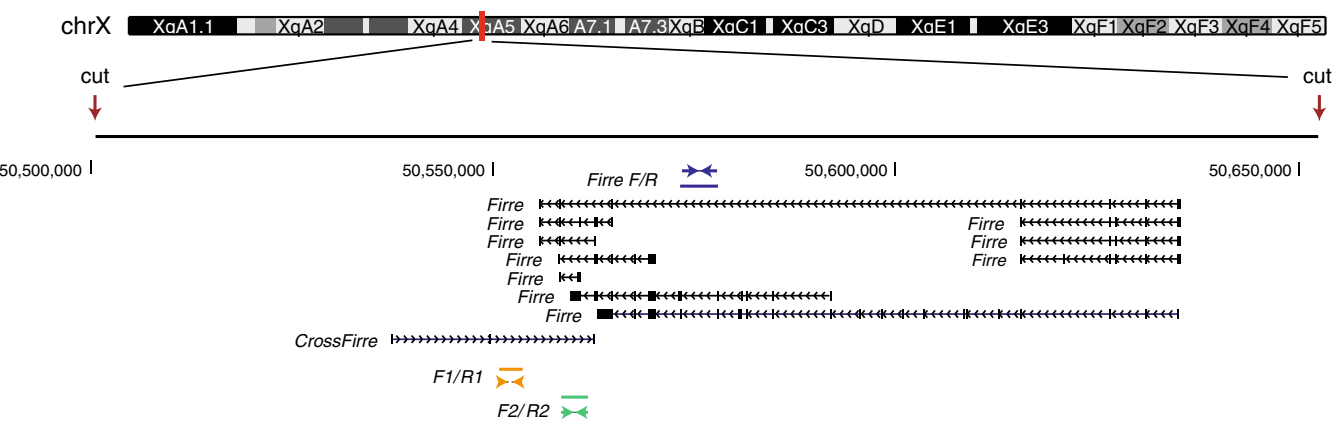

b

RT-PCR

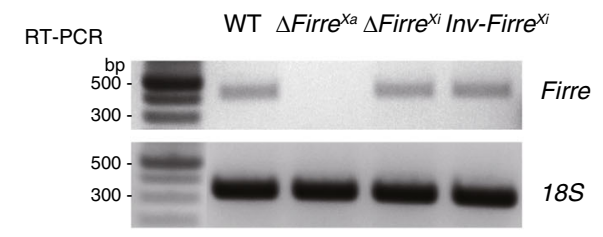

d

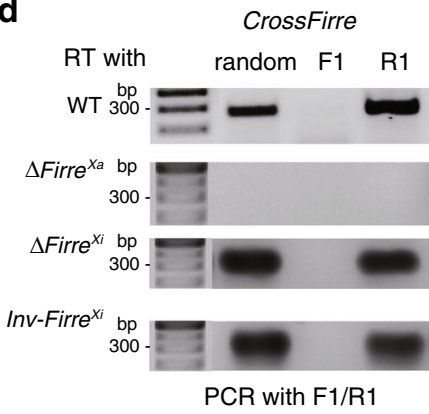

Firre

$8 S$

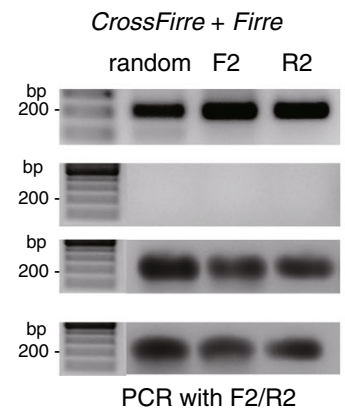

C

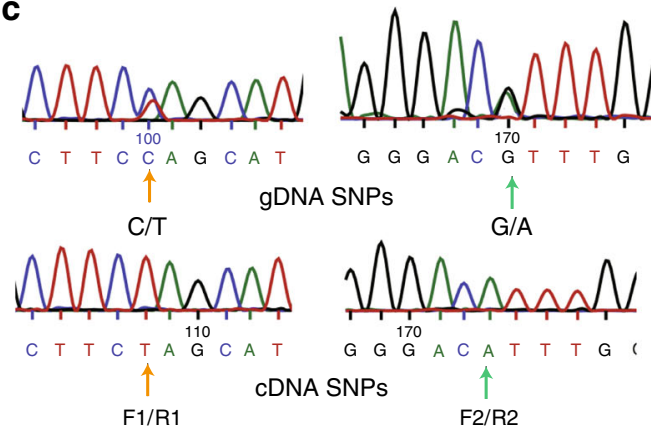

Fig. 1 Firre and CrossFirre are expressed from the Xa. a Genomic location of Firre and CrossFirre (Gm35612) on the mouse X chromosome (UCSC mm10 build 38 browser tracks). Note that Firre has multiple alternative transcripts. The locations of the CRISPR guide RNAs used to edit the locus (cut), and of the RT-PCR primer pairs to specifically detect Firre expression (F/R), strand-specific expression of CrossFirre (F1/R1), or strand-specific expression in a region of overlap between Firre and CrossFirre (F2/R2) are indicated. b RT-PCR analysis using the F/R primer pair detects Firre expression in WT, $\Delta$ Firre $\mathrm{Xi}$, and InvFirre $\mathrm{Xi}_{\mathrm{i}}$, but not in $\Delta$ Firre $^{\mathrm{X}}$. Firre expression was measured in $n=3$ biologically independent samples per cell type. c Sanger sequencing analyses of a CrossFirre region (F1/R1) and of a region of overlap between Firre and CrossFirre (F2/R2) confirm heterozygosity of SNPs (BL6 on the Xi and spretus on the $\mathrm{Xa}$ ) in each region assayed. Genomic DNA (gDNA) shows heterozygosity at the SNPs, while cDNA only shows expression from the spretus SNP (Xa). d Strand-specific analysis of CrossFirre and Firre was done using reverse transcription using either random primers, or F1, R1, F2, R2 primers, followed by PCR using F1/R1 or F2/R2 primer pairs. Firre and CrossFirre expression was measured in $n=3$ biologically independent samples per cell type. Source data are provided as a Source Data file.

the Xi in $\Delta$ Firre ${ }^{\mathrm{Xa}}$ (Fig. 2d). The loss of H3K27me3 in mutant cells was quantified by counting unique reads mapped to the $\mathrm{Xa}$, $\mathrm{Xi}$, and autosomes. In $\mathrm{WT}$ the $\mathrm{Xi} / \mathrm{Xa}$ read ratio was 2.45 , reflecting the characteristic H3K27me3 enrichment on the Xi. In contrast, this ratio was only 1.60 in $\Delta F_{i r r e}{ }^{\mathrm{Xa}}$, representing a significant decrease on the Xi (Fig. 2d). The BL6/spretus read ratios for autosomes in WT and $\Delta$ Firre ${ }^{\mathrm{Xa}}$ remained similar, 1.04 and 1.14, respectively. The $34 \%$ percent decrease in the level of H3K27me3 on the Xi as measured by ChIP-seq is lower than that measured by immunostaining (89\%), which is expected based on known differences between methodologies. While immunostaining captures condensation of the $\mathrm{Xi}$ and thus results in many nuclei with a visible H3K27me3 cluster, ChIP-seq reveals uneven distribution of H3K27me3 along the Xi and thus an apparently lesser enrichment ${ }^{37}$. In fact, the $34 \%$ change in $\mathrm{H} 3 \mathrm{~K} 27 \mathrm{me} 3$ we measured by ChIP-seq in $\Delta$ Firre ${ }^{\mathrm{Xa}}$ versus WT is similar to that reported between undifferentiated and differentiated mouse ES cells, in which the onset of XCI causes only a $40 \%$ increase in H3K27me3 level as measured by ChIP-seq, while over $90 \%$ of nuclei acquire a visible H3K27me3 cluster, as determined by immunostaining ${ }^{37}$.
Next, we calculated allelic proportions of SNP read coverage for each H3K27me3 peak covered by at least five SNP reads. In both WT and $\Delta$ Firre $^{\mathrm{Xa}}$ the distribution of allelic proportions (spretus/(spretus + BL6)) for the autosomes centered close to the anticipated 0.5 , reflecting a similar enrichment between alleles (Fig. 2e). In contrast, the distribution of allelic proportions for the $\mathrm{X}$ chromosomes $(\mathrm{Xa} /(\mathrm{Xa}+\mathrm{Xi}))$ centered at $\sim 0.35$ in $\mathrm{WT}$, consistent with $\mathrm{H} 3 \mathrm{~K} 27 \mathrm{me} 3$ enrichment on the $\mathrm{Xi}$, but was markedly shifted to higher values ( 0.5) in $\Delta$ Firre $^{\mathrm{Xa}}$, supporting the $34 \%$ decrease in H3K27me3 on the Xi (Fig. 2e). Heatmaps and metagene plots of allelic ChIP-seq data further demonstrate a dramatic loss of $\mathrm{H} 3 \mathrm{~K} 27 \mathrm{me} 3$ around the transcription start site and throughout the body of X-linked genes in mutant cells (Fig. 2f, g). LINE and SINE repeats also show lower H3K27me3 on the Xi in $\Delta$ Firre $^{\mathrm{Xa}}$ (Supplementary Fig. 2c). Finally, we confirmed a decrease in $\mathrm{H} 3 \mathrm{~K} 27 \mathrm{me} 3$ in $\Delta$ Firre $^{\mathrm{Xa}}$ using CUT\&RUN in a separate study ${ }^{38}$.

Next, we investigated SUZ12, a subunit of the PRC2 complex, using CUT\&RUN, which showed a decrease on the Xi in $\Delta$ Firre $^{\mathrm{Xa}}$. Again, the distribution of allelic proportions $(\mathrm{Xa} /(\mathrm{Xa}+\mathrm{Xi}))$ for each SUZ12 peak showed a pronounced shift toward higher values 
a

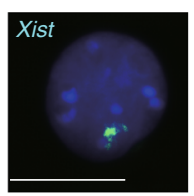

WT

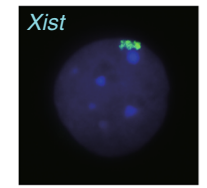

$\Delta$ Firre $^{X a}$

b

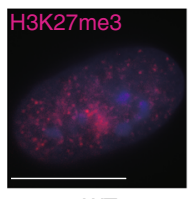

WT

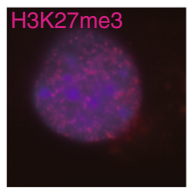

$\Delta$ Firre $^{X_{a}}$

C

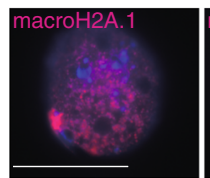

WT

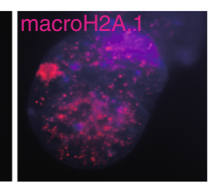

$\Delta$ Firre $^{X_{a}}$

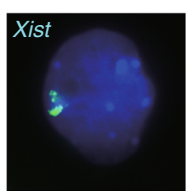

$\Delta$ Firre $^{x_{i}}$

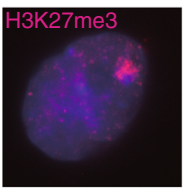

$\Delta$ Firre $^{X_{i}}$

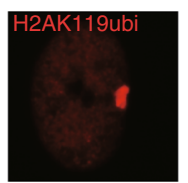

WT

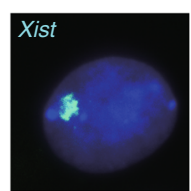

Inv-Firre ${ }^{X_{i}}$

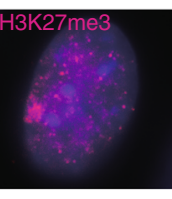

Inv-Firre ${ }^{X_{i}}$

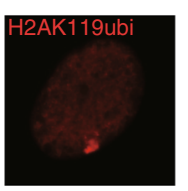

$\Delta$ Firre $^{x a}$
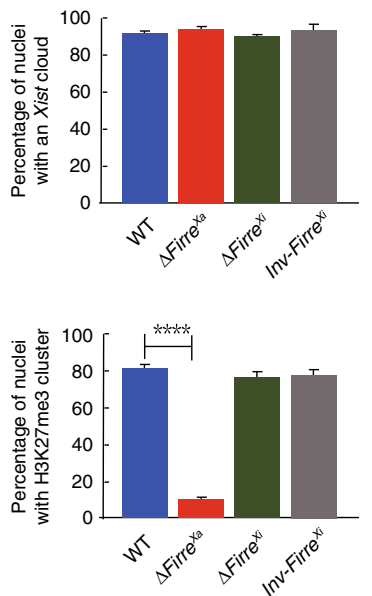

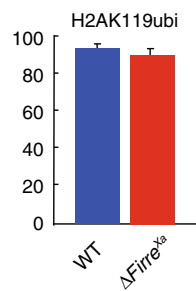

f

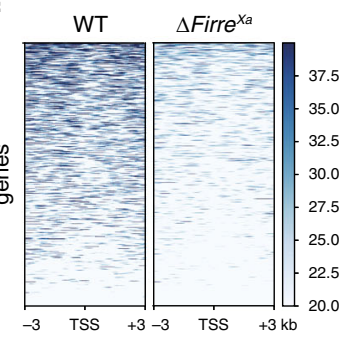

g
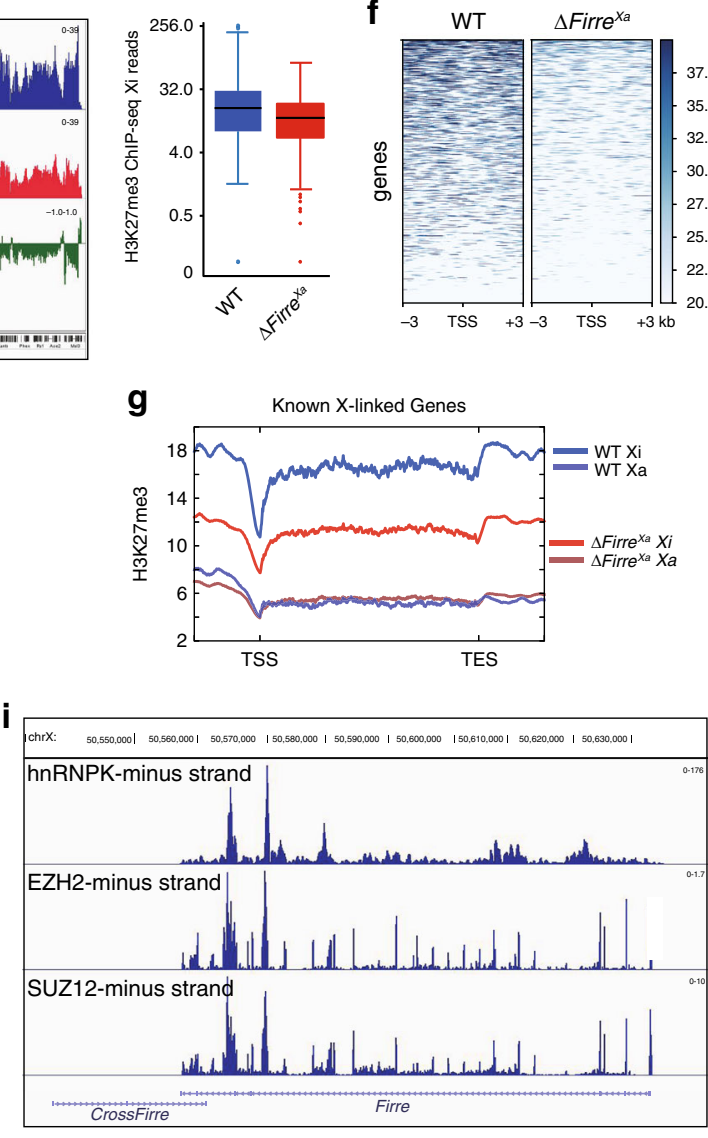

SUZ12, and with hnRNPK, a protein that recruits the noncanonical PCGF3/5-PRC1 (Fig. 2i; Supplementary Data 5) ${ }^{39,40}$. Analysis of data obtained by CLIP-seq and PAR-CLIP, two UVcrosslinking-based methods for highly accurate mapping of RNAprotein interactions, confirmed Firre RNA interactions with EZH2 and detected interactions with JARID2 and RBFOX2, two $(\sim 0.75)$ for the $\mathrm{X}$ chromosomes in $\Delta$ Firre $^{\mathrm{Xa}}$ versus $\mathrm{WT}$, while allelic proportions for the autosomes (0.5) did not change (Fig. 2h). Reanalysis of published datasets of RNA/protein interactions in mouse cells confirmed Firre RNA interactions with PRC components. Analysis of RIP-seq data showed Firre RNA interactions with two components of PRC2, EZH2 and

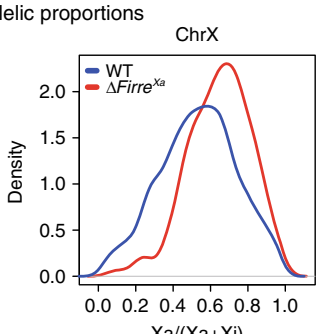


Fig. 2 Firre RNA acts in trans to maintain H3K27me3 on the Xi. a-c A total of $>300$ Patski nuclei were scored per cell type over 3 independent experiments; significance was determined by one-sided Fisher exact test; bar plots are presented as mean values \pm SEM; scale bars represent $10 \mu \mathrm{m}$. a Examples of nuclei after Xist RNA-FISH (green) and Hoechst 33342 staining (blue). The bar plot shows no significant differences among cell lines. b Examples of nuclei after H3K27me3 immunostaining (red) and Hoechst 33342 staining (blue). The bar plot shows significantly fewer nuclei with a H3K27me3 cluster in $\Delta$ Firre ${ }^{X a}$ versus WT ( $p$ value $=4.63688 \mathrm{e}-94$ ), but no change in $\Delta$ Firre ${ }^{X i}$ nor InvFirre ${ }^{X i}$. c Examples of nuclei after macroH2A.1 or H2AK119ubi (red) immunostaining and Hoechst 33342 staining (blue). The bar plots show no significant differences between cell types. d Profiles of H3K27me3 ChIP-seq reads along the Xi in WT (blue), $\Delta$ FirreXa (red), and $\log _{2}$ ratio $\Delta$ FirreXa/WT (green). Box plots $\left(\log _{2}\right.$ ) of H3K27me3 ChIP-seq reads in $100 \mathrm{bp} \mathrm{Xi}$ bins show a significantly lower median in $\Delta$ Firre $\mathrm{Xa}^{(\mathrm{red})}$ versus WT (blue) (Wilcoxon test: $p$ value $=2.2 \mathrm{e}-16$ ). The boxes demarcate the interquartile range (IQR) with median; whiskers \pm 1.5 times the IQR; outliers plotted as points. e Density histograms of the distribution of allelic proportions $(\mathrm{Xa} /(\mathrm{Xa}+\mathrm{Xi})$ ) of H3K27me3 peaks show a shift for the $\mathrm{X}$ chromosomes due to lower H3K27me3 on the $\mathrm{Xi}$ in $\Delta$ Firre $\mathrm{Xa}$ (red) compared to WT (blue) (Wilcoxon test: $-\log 10 \mathrm{P}=$ inf). $\mathbf{f}$ Heatmaps of H3K27me3 ChIP-seq reads $3 \mathrm{~kb}$ around transcription start sites (TSS) of genes on the $X_{i}$ in $\Delta F$ irre $X_{a}$ versus WT. g. Metagene plots of average H3K27me3 occupancy at X-linked genes ((TSS to termination site (TES), not at scale)) in $\Delta$ Firre ${ }^{X a}$ (Xi red Xa pink) versus WT (Xi blue, Xa purple). $\mathbf{h}$ Density histograms of the distribution of allelic proportions $(\mathrm{Xa} /(\mathrm{Xa}+\mathrm{Xi}))$ of SUZ12 peaks show a shift for the $\mathrm{X}$ chromosomes due to lower SUZ12 on the Xi in $\Delta$ Firre ${ }^{X}$ (red) versus WT (blue) (Wilcoxon test: $-\log 10 P=20.98$ ). $\mathbf{i}$ Genome tracks demonstrating interactions between hnRNPK, EZH2, and SUZ12 with Firre RNA based on RIP-seq data in trophoblast and embryo stem cells 39,40 .

\begin{tabular}{|c|c|c|}
\hline Cell lines & Genotype & Percent nuclei \\
\hline \multirow[t]{3}{*}{$\begin{array}{l}\text { Patski cells derived from kidney } \\
\text { from an } \mathrm{F} 1 \text { embryo (BL6 } \mathrm{Hprt}^{B M 3} \times \\
\text { spretus) }\end{array}$} & $\begin{array}{l}\text { WT } \\
\Delta \text { Firre } \mathrm{Xa}^{\mathrm{Xa}} \\
\Delta \text { Firre }{ }^{\mathrm{Xa}} \\
\text { +mtransgene }\end{array}$ & $\begin{array}{l}83 \% \\
9 \% \\
38 \%\end{array}$ \\
\hline & $\begin{array}{l}\Delta \text { Firre }^{\mathrm{Xa}} \\
\text { +htransgene }\end{array}$ & $21 \%$ \\
\hline & $\begin{array}{l}\Delta \text { Firre }^{\mathrm{Xi}} \\
\text { InvFirre } \mathrm{Xi} \\
\text { Firre shRNA KD } \\
\text { Firre siRNA KD } \\
\text { Firre si/ } \\
\text { shRNA KD }\end{array}$ & $\begin{array}{l}77 \% \\
79 \% \\
33 \% \\
27 \% \\
15 \%\end{array}$ \\
\hline $\begin{array}{l}\text { Primary MEFs derived from an F1 } \\
\text { embryo (BL6 } \times \text { spretus) } \\
\text { Primary MEFs derived from an F1 } \\
\text { embryo with skewed } \mathrm{XCl} \text { (BL6 } \\
\text { Xist }^{\Delta} \times \text { spretus) }\end{array}$ & $\begin{array}{l}\text { Control } \\
\text { Firre KD } \\
\text { Control } \\
\text { Firre KD }\end{array}$ & $\begin{array}{l}79 \% \\
43 \% \\
80 \% \\
45 \%\end{array}$ \\
\hline \multirow{7}{*}{$\begin{array}{l}\text { Primary MEFs derived from an F1 } \\
\text { embryo (BL6 } \times \text { castaneus) } \\
\text { Primary MEFs derived from a Firre } \\
\text { KO mouse model, with or without } \\
\text { induction of Firre by doxycycline }\end{array}$} & $\begin{array}{l}\text { Control } \\
\text { Firre KD }\end{array}$ & $\begin{array}{l}78 \% \\
47 \%\end{array}$ \\
\hline & $\begin{array}{l}\text { Firre }^{+/+} \text {control } \\
\text { Firre }^{+/-} \\
\text {heterozygote }\end{array}$ & $\begin{array}{l}58 \% \\
43 \%\end{array}$ \\
\hline & $\begin{array}{l}\text { Firre }^{-1-} \\
\text { homozygote }\end{array}$ & $41 \%$ \\
\hline & $\begin{array}{l}\text { Firre }^{+/-} \text {tg;rtTA; } \\
- \text { Dox }\end{array}$ & $52 \%$ \\
\hline & $\begin{array}{l}\text { Firre }^{+/-} \text {tg;rtTA; } \\
+ \text { Dox }\end{array}$ & $91 \%$ \\
\hline & $\begin{array}{l}\text { Firre }^{-/-} \text {tg;rtTA; } \\
- \text { Dox }\end{array}$ & $49 \%$ \\
\hline & $\begin{array}{l}\text { Firre }^{-/-} \text {tg; } \\
\text { rtTA; +Dox }\end{array}$ & $83 \%$ \\
\hline \multirow{6}{*}{$\begin{array}{l}\text { Tissues derived from a Firre KO } \\
\text { mouse model }\end{array}$} & Firre $^{+/+}$brain & $71 \%$ \\
\hline & Firre $^{-/-}$brain & $70 \%$ \\
\hline & Firre $^{+/}+$liver & $67 \%$ \\
\hline & Firre $^{-/-}$liver & $69 \%$ \\
\hline & Firre $^{+/+}$kidney & $56 \%$ \\
\hline & Firre $^{-/-}$kidney & $58 \%$ \\
\hline
\end{tabular}

The table lists each cell line, including its origin, its genotype, and the percentage of nuclei with a H3K27me3 cluster on the Xi.

proteins known to help recruit PRC2 to chromatin, and with CBX7, a component of the canonical PRC1 complex implicated in H3K27me3 deposition ${ }^{41-45}$ (Supplementary Data 5).
We conclude that Firre RNA transcribed from the Xa specifically helps target PRC1 and PRC2 complexes to the Xi for maintenance of $\mathrm{H} 3 \mathrm{~K} 27 \mathrm{me} 3$, consistent with a trans-acting effect in Patski cells. In support we find that several proteins implicated in the PRC complexes are Firre RNA interactors.

Dose-dependent and cell-type-specific effects of Firre. To quantify the effects of Firre, we knocked it down in a dosedependent manner using shRNA and siRNA treatments of an independent Patski isolate to achieve $\mathrm{KD}$ levels ranging from 43, 28 , and $10 \%$ of WT. This caused a parallel reduction in percentages of nuclei with a $\mathrm{H} 3 \mathrm{~K} 27 \mathrm{me} 3$ cluster to $33 \%, 27 \%$, and $15 \%$, respectively, versus $83 \%$ in WT $(p<0.0001)$ (Fig. 3a; Table 1$)$. Importantly, this reveals a dose-dependent effect of Firre RNA on H3K27me3 enrichment on the Xi (Fig. 3a). Concomitantly, a dose-dependent increase in CrossFirre RNA inversely correlates to the amount of Firre RNA, consistent with Firre repressing the antisense transcript (Fig. 3b).

A separate KD to deplete Firre RNA to $33 \%$ of WT in primary mouse embryonic fibroblasts (MEFs) independently derived from a BL6 $\times$ spretus F1 embryo with random XCI significantly decreased the percentage of nuclei with a H3K27me3 cluster to $43 \%$, versus $79 \%$ in controls $\left(p<10^{-4}\right)$ (Fig. 3c; Table 1). To exclude species-specific effects, we tested MEFs from a BL6 $\times$ spretus F1 embryo with an XCI pattern opposite to that in Patski cells (i.e., the $\mathrm{Xi}$ is from spretus), and MEFs from a BL6 $\times$ castaneus F1 embryo. After KD in these lines Firre RNA level was lowered to 21 and $15 \%$ of $\mathrm{WT}$, causing a significant reduction to 45 and $47 \%$ of nuclei with a H3K27me3 cluster, compared to 80 and $78 \%$ in controls, respectively $\left(p<10^{-4}\right)$ (Fig. 3c; Supplementary Fig. 2d; Table 1). No significant change in CrossFirre was found in those lines (Supplementary Fig. 2e). We then examined cells and tissues from a Firre KO mouse model ${ }^{32,33}$. MEFs from heterozygous (Firre ${ }^{+-}$) and homozygous (Firre ${ }^{-l-}$ ) KO female embryos showed a modest reduction to 43 and $41 \%$ of nuclei with a H3K27me3 cluster, respectively, compared to $58 \%$ in a control littermate $\left(\right.$ Firre $\left.^{+/+}\right)$(Fig. 3d; Table 1). H3K27me3 immunostaining in liver, kidney, and brain sections derived from Firre ${ }^{-/-}$ female mice showed no significant decrease in nuclei with a cluster (Fig. 3d; Table 1).

Next, we performed H3K27me3 ChIP-seq analysis of sorted CLPs and CD $8+\mathrm{T}$ cells derived from Firre ${ }^{-1-} \mathrm{KO}$ female mice. Surprisingly, we found a striking loss of H3K27me3 in a $\sim 26 \mathrm{Mb}$ (ChrX: 36-62 Mb) region centered around the Firre locus, together with a slight decrease across the entire $\mathrm{X}$ chromosome, but not the autosomes (Fig. 3e, Supplementary Fig. 3a, b). 
a

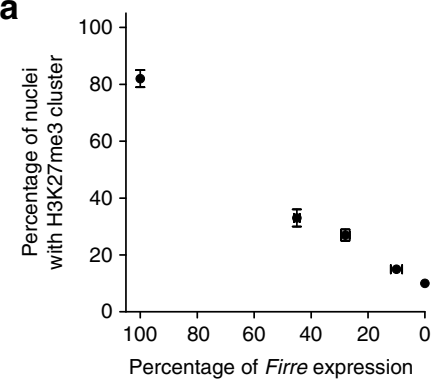

b

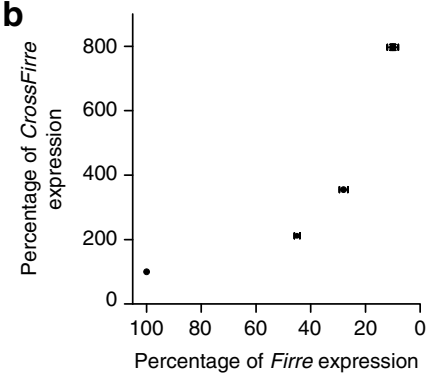

C

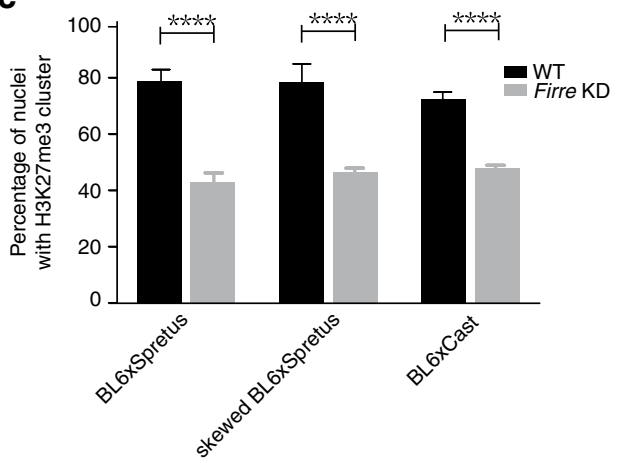

d

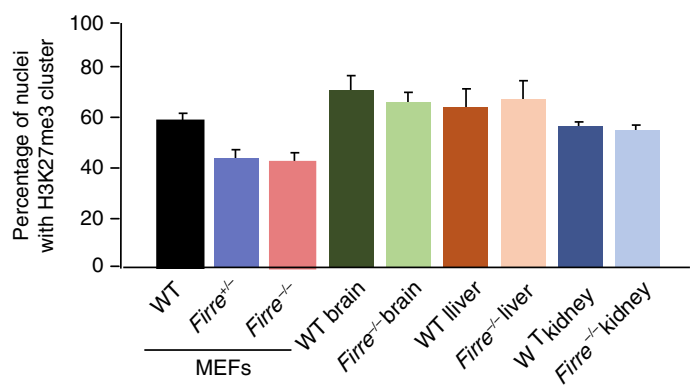

$\mathbf{e}$

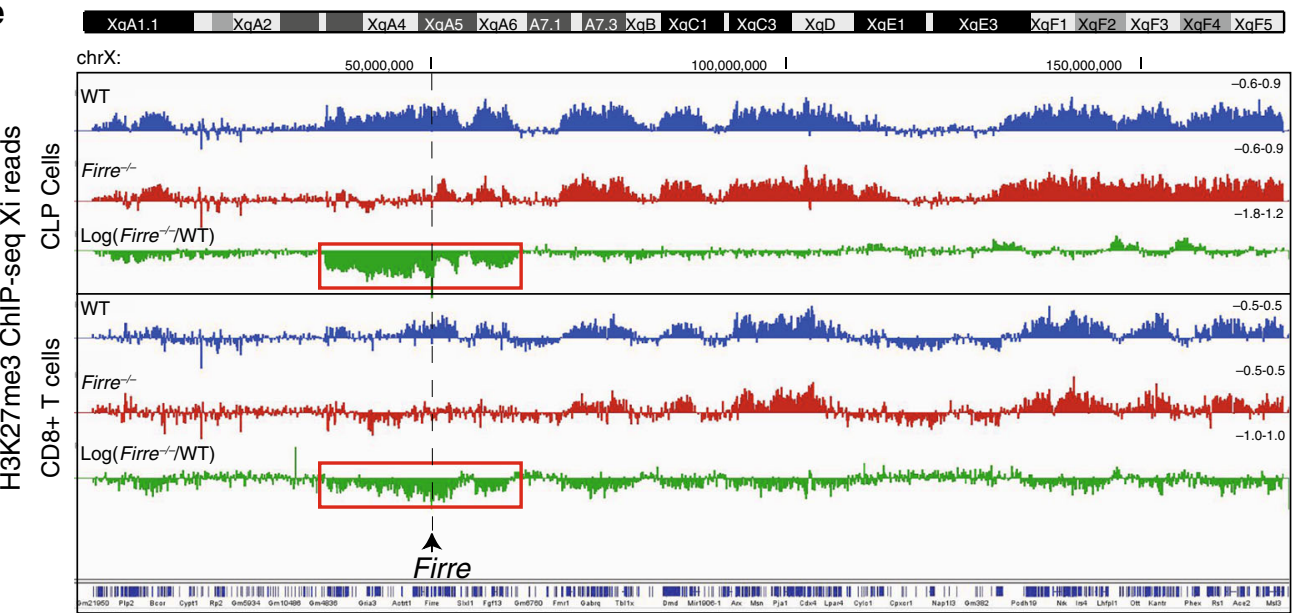

f

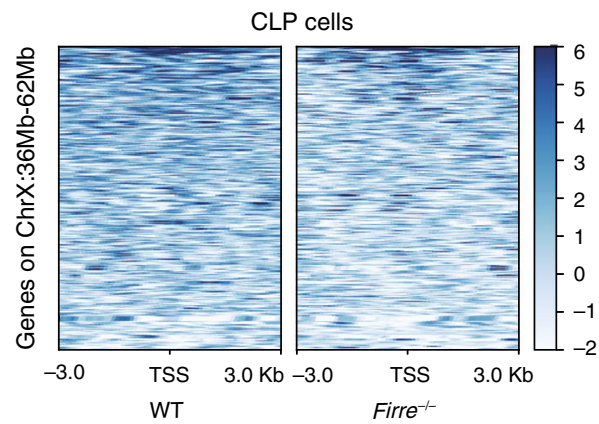

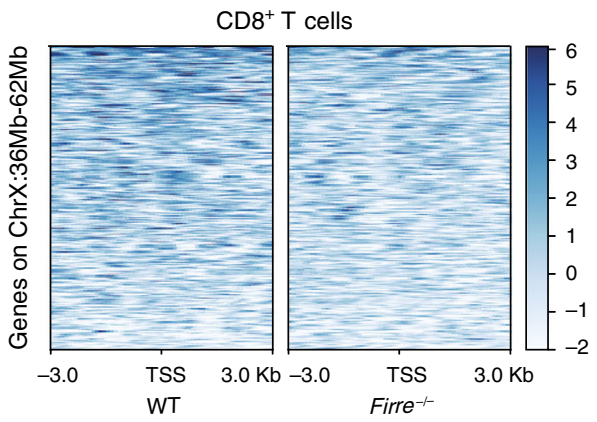

exhibiting haploinsufficiency effects in human (e.g., $G P C 3^{49}$ ) (Supplementary Data 6). However, no specific expression changes have been reported for any of these genes in CLPs from KO mice $^{33}$.

In summary, we found a dose-dependent effect of Firre RNA in maintenance of $\mathrm{H} 3 \mathrm{~K} 27 \mathrm{me} 3$ on the $\mathrm{Xi}$ in several types of fibroblasts. Lack of detectable changes in three tissues from Firre KO mice suggests that the role of Firre may be cell-type- and Firre contains 266 curated genes, 15 involved in immunity (e.g., Nkap, Sash3, Atp11c, Sh2d1a, Elf $4^{46-48}$ ), and 15 potentially

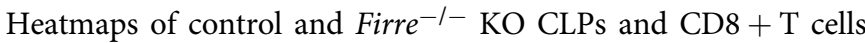
showed a loss of $\mathrm{H} 3 \mathrm{~K} 27 \mathrm{me} 3$ around the transcription start site of genes located within this $26 \mathrm{Mb}$ region (Fig. 3f, Supplementary Data 6). Although we assume that this local loss of $\mathrm{H} 3 \mathrm{~K} 27 \mathrm{me} 3$ affects the $\mathrm{Xi}$ and not the $\mathrm{Xa}$, allelic analysis was not possible 
Fig. 3 Dose-dependent effects of Firre RNA on H3K27me3 on the Xi in cell lines and in vivo. a Percentage of nuclei with a H3K27me3 cluster after KD in Patski cells relative to Firre expression level measured by $q R T-P C R$ in $n=3$ biologically independent samples. Data presented as mean values \pm SEM. $\mathbf{b}$ The level of Crossfirre expression is inversely related to that of Firre after Firre KD. Expression measured by qRT-PCR in $n=3$ biologically independent samples. Data presented as mean values \pm SEM. c, d A total of $>300$ Patski nuclei were scored per cell type over three independent experiments; significance was determined by one-sided Fisher exact test; bar plots are presented as mean values \pm SEM; scale bars represent $10 \mu \mathrm{m}$. c Bar plots show significantly fewer H3K27me3 clusters after Firre KD in nuclei from three primary MEFs compared to mock treatment ( $p$ value $=2.89075 \mathrm{e}-12,1.41519 \mathrm{e}-32$, and $8.95838 \mathrm{e}-10$, respectively). Primary MEFs were derived from a (BL6 $\times$ spretus) embryo with a spretus $\mathrm{Xi}$, and from embryos either (BL6 $\times$ spretus) or (BL6 $\times$ castaneus) with random $\mathrm{XCl}$ (Table1). d Bar plots show a lower (but not significantly) percentage of H3K27me3 cluster in nuclei from MEFs derived from Firre ${ }^{+/-}$and Firre $^{-1-} \mathrm{KO}$ embryos than in controls ( $p$ value $=0.0774$ and 0.0567 , respectively). No significant differences were found in brain, liver, and kidney from Firre KO mice compared to WT ( $p$ value $=0.9127,0.8796$, and 0.762 , respectively). e Profiles of H3K27me3 ChIP-seq reads along the X chromosomes from CLPs and CD8 + T cells from WT (blue), Firre ${ }^{-/-}$(red), and $\log _{2}$ ratio Firre ${ }^{-/-}$/WT (green) show a significant decrease covering $\sim 26 \mathrm{Mb}$ around the Firre locus in mutant CLPs, and to a lesser extent CD8 + T cells (Supplementary Data 6). $\mathbf{f}$ Heatmaps of H3K27me3 ChIP-seq reads located 3 kb around the transcription start sites (TSS) of X-linked genes that map within the $26 \mathrm{Mb}$ region around Firre in WT and Firre-/- CLPs and CD8 + T cells.

tissue-specific, as proposed for other $\operatorname{lncRNAs}{ }^{50}$. Consistent with the role of Firre in hematopoiesis, local changes were observed in CLPs and CD8 + T cells around the Firre locus, revealing a celltype-specific role in cis-maintenance of $\mathrm{H} 3 \mathrm{~K} 27 \mathrm{me} 3^{33}$.

Rescue of H3K27me3 on the Xi by Firre/FIRRE cDNA transgenes. To confirm a causative role of Firre RNA on H3K27me3 enrichment on the $\mathrm{Xi}, \Delta$ Firre $^{\mathrm{Xa}}$ cells were transfected with a mouse Firre cDNA transgene that lacks the first five 5' exons of Firre and is expressed from a CMV promoter (Supplementary Fig. 1b). By RNA-seq Firre expression was restored to a nearnormal level after transfection. Ectopic expression of the mouse cDNA in $\triangle$ Firre $\mathrm{Xa}^{\mathrm{Xa}+\mathrm{mtransgene}}$ cells rescued the presence of a H3K27me3 cluster from 9 to $38 \%$ of nuclei, supporting a transacting role (Fig. 4a, b, Table 1). This partial rescue is not readily explained by heterogeneous transgene levels among cells, since a cloned transgenic line with stable high Firre expression rescued to a similar level (34\%). Firre RNA-FISH in this clone showed association of the lncRNA to the Xist cloud in $15 \%$ of cells, consistent with partial rescue (Fig. 4c). Incomplete rescue is likely due to the partial cDNA transgene that may lack functional RNA motifs, isoforms, and regulatory elements (Supplementary Fig. 1b). Interestingly, we also observed partial rescue after ectopic expression of a human FIRRE cDNA in $\triangle F i r r e^{\mathrm{Xa}+\mathrm{htrans}-}$ gene cells (Fig. 4a, b; Table 1). Next, we examined Firre ${ }^{+/-}$and Firre $^{-1-}$ MEFs from KO mice with an ectopic doxycycline (DOX)-inducible plasmid copy of a complete Firre cDNA integrated in the genome. After Firre expression induction (10-20 fold) a strong increase to 91 and $83 \%$ of nuclei with a H3K27me3 cluster was found compared to 43 and $41 \%$ in noninduced $\mathrm{KO}$ cells, respectively (Fig. 4d; Table 1).

We conclude that loss of $\mathrm{H} 3 \mathrm{~K} 27 \mathrm{me} 3$ on the $\mathrm{Xi}$ in mutant cells is rescuable by a cDNA transgene, supporting Firre trans-acting role. Surprisingly, a human FIRRE transgene can also rescue to some extent, suggesting functional compatibility between species despite sequence divergence. Results of induction of a transgene in the mouse $\mathrm{KO}$ model further support a dose-dependent transeffect of Firre RNA on H3K27me3 enrichment on the Xi.

Firre acts in trans to maintain nuclear location of the $\mathrm{Xi}$. We next examined the effects of allelic Firre mutations on the $\mathrm{Xi}$ location relative to the nucleolus and lamina. In WT, $\Delta$ Firre $^{\mathrm{Xi}}$, and InvFirre ${ }^{\mathrm{Xi}} \mathrm{H} 3 \mathrm{~K} 27 \mathrm{me} 3$ and nucleophosmin (NPM1) immunostaining was used to locate the $\mathrm{Xi}$ and the nucleoli, respectively (Fig. 5a). Since the H3K27me3 cluster is compromised in $\Delta$ Firre ${ }^{\mathrm{Xa}}$, Xist RNA-FISH was applied in combination with NPM1 immunostaining. The Xi location was scored in WT nuclei as adjacent to the nuclear periphery $(70 \%)$, the nucleolus surface $(50 \%)$, or neither $(8 \%)$ (Fig. 5 b). Note that in $28 \%$ of nuclei the Xi was close to both the periphery and the nucleolus. Loss of Firre RNA in $\triangle$ Firre $^{\mathrm{Xa}}$ caused significant reductions in Xi-nuclear periphery and $\mathrm{Xi}$-nucleolus associations to 20 and $22 \%$ of nuclei, respectively $(p<0.0001)$ (Fig. 5b). Importantly, ectopic expression of a cDNA transgene in $\Delta$ Firre $\mathrm{Xa}+$ mtransgene significantly (but not completely) rescued these associations (Fig. 5b). Deletion or inversion of Firre on the Xi did not alter its location (Fig. 5a, b). In primary MEFs independently derived from a BL6 $\times$ spretus F1 mouse with random XCI Firre KD also caused a decrease in Xi-nuclear periphery and $\mathrm{Xi}$-nucleolus associations (Fig. 5c).

CTCF has been implicated in nucleolus association of genomic regions, and we have previously shown that $C t c f \mathrm{KD}$ reduces $\mathrm{Xi}$ nucleolus associations in Patski cells ${ }^{20,51}$. Here, we profiled allelic CTCF binding by CUT\&RUN, which demonstrated a loss of CTCF binding on the $\mathrm{Xi}$ in $\triangle$ Firre $^{\mathrm{Xa}}$ (Fig. $5 \mathrm{~d}$ ). The distribution of allelic proportions $(\mathrm{Xa} /(\mathrm{Xa}+\mathrm{Xi}))$ for $\mathrm{CTCF}$ peaks on the $\mathrm{X}$ chromosomes showed a pronounced shift toward higher values $(\sim 0.85)$ in $\Delta$ Firre $^{\mathrm{Xa}}$ compared to WT $(\sim 0.65)$, while allelic proportions (spretus/(spretus + BL6)) for the autosomes were close to the anticipated 0.5 (Fig. $5 \mathrm{~d}$ ). Thus, while there is less CTCF binding on the $\mathrm{Xi}$ versus $\mathrm{Xa}$ in $\mathrm{WT}$ as expected, CTCF binding is even lower in $\Delta$ Firre $^{\mathrm{Xa}}$ (Fig. 5e). In $\Delta$ Firre $^{\mathrm{Xa}+\text { mtransgene }}$ the distribution of allelic proportions for the $\mathrm{X}$ chromosomes becomes binomial, indicating partial restoration of CTCF on the Xi (Fig. 5d).

Taken together, our results show that Firre RNA transcribed from the Xa or from an ectopic cDNA transgene can influence in trans the $\mathrm{Xi}$ location within the nucleus. Our results further suggest a potential cooperation between Firre RNA and CTCF in maintenance of Xi location.

Loss of Firre affects gene expression in Patski cells. Next, we examined changes in total gene expression (autosomal and Xlinked genes without allele discrimination) in $\Delta$ Firre $^{\mathrm{Xa}}$. About 11 and $14 \%$ of genes with expression $\geq 1 \mathrm{TPM}$ in at least one condition were upregulated and downregulated in $\Delta$ Firre $^{\mathrm{Xa}}$ versus WT, respectively, (Supplementary Data 7 and 8). A large proportion (46 and $40 \%$, respectively) of dysregulated genes were rescued in $\Delta$ Firre $^{\mathrm{Xa}+\text { mtransgene }}$ (Fig. 6a, b, Supplementary Fig. 4a, Supplementary Data 7). To rule out effects of aneuploidy between cell lines results were confirmed for genes located on diploid chromosomes in WT and $\Delta$ Firre $^{\mathrm{Xa}}$ (Supplementary Fig. 4b, Supplementary Data 9). GO analysis show that the top 20 GO terms for genes upregulated in $\Delta$ Firre $^{\mathrm{Xa}}$ and rescued in $\Delta F i r r e^{\mathrm{Xa}+\text { mtransgene }}$ are related to cell cycle, DNA replication, chromosome segregation, and immune cell function, while downregulated genes are implicated in development, differentiation, and metabolism (Fig. 6a, b; Supplementary Data 10). In human HEK293 cells FIRRE RNA KD resulted in dose-dependent cell death, consistent with a role in cell growth and survival (Supplementary Fig. 4c). 
a

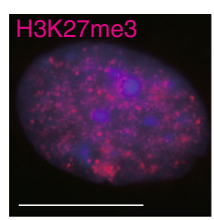

WT

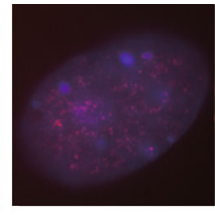

$\Delta$ Firre $^{x_{a}}$

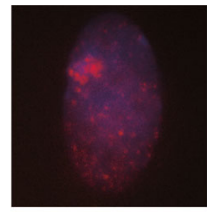

$\Delta$ Firre $^{X_{a}}$

+mouse cDNA

transgene

c

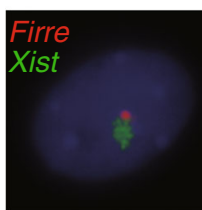

$\cdot+\cdot$

$\Delta$ Firre $^{\text {xa }}$

+mouse cDNA

transgene b

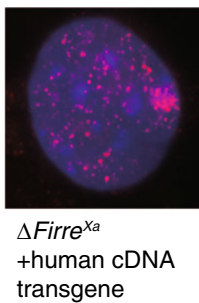

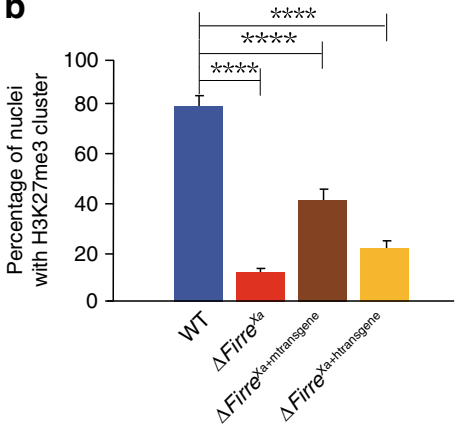

d

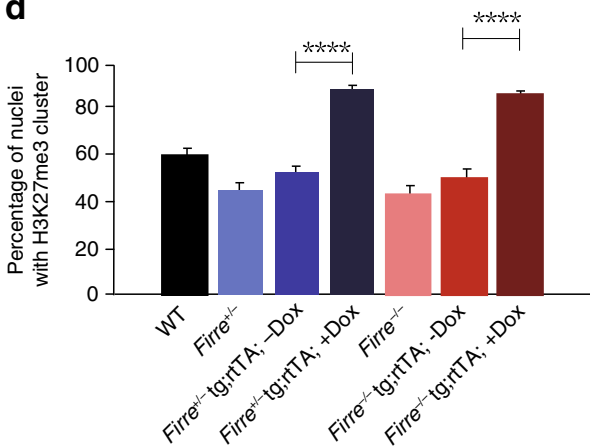

Fig. 4 Ectopic expression of Firre/FIRRE RNA partially restore H3K27me3 on the Xi. a Examples of nuclei after H3K27me3 immunostaining (red) and Hoechst 33342 staining (blue) in WT, $\triangle$ Firre ${ }^{\mathrm{Xa}}$, and $\Delta$ Firre $^{\mathrm{Xa}}$ transfected with a mouse Firre transgene ( $\triangle$ Firre $\left.{ }^{\mathrm{X}}+\mathrm{mtransgene}\right)$ or a human FIRRE transgene ( $\triangle$ Firre $\mathrm{Xa}_{\mathrm{a}}$ htransgene). b The bar plot shows a significantly higher percentage of nuclei with a H3K27me3 cluster in cells with a mouse or human Firre/FIRRE transgene, compared to $\Delta$ Firre ${ }^{X a}$ ( $p$ value $=2.8075$ - 16 and 0.0000134599 , respectively). c Examples of nuclei after RNA-FISH for Xist (green) and Firre (red) in a $\Delta$ Firre $\mathrm{Xa}+$ mtransgene cell clone with high expression of the mouse transgene. The upper two nuclei show association between Firre and Xist signals (seen in $15 \%$ of nuclei), and the lower nuclei, lack of association. $\mathbf{d}$ The bar plot shows the percentage of nuclei with a H3K27me3 cluster in MEFs derived from Firre ${ }^{+/-}$and Firre ${ }^{-/-}$females that harbor a doxycycline (Dox) inducible transgene (Firre ${ }^{+/-}$tg;rtTA; - Dox; Firre ${ }^{+/-}$tg;rtTA; +Dox; Firre ${ }^{-/-}$tg; trTA; $^{-}$ -Dox; Firre ${ }^{-/-}$tg;rtTA; +Dox), compared to WT and mutants. The percentage of nuclei with a H3K27me3 cluster increases significantly in Firre ${ }^{+/-}$tg; rtTA and Firre ${ }^{-/-}$tg;rtTA MEFs after addition of doxycycline (Dox + ) ( $p$ value $=4.31567 \mathrm{e}-30$ and 3.51193e-20, respectively). A total of $>300$ nuclei scored for the presence of a H3K27me3 cluster (a, b, d), or for Xist and Firre RNA-FISH signals (c) per cell type over three independent experiments; significance was determined by one-sided Fisher exact test; bar plots are presented as mean values \pm SEM; scale bars represent $10 \mu \mathrm{m}$.

To determine whether gene expression was disrupted on the Xi upon loss of Firre RNA we evaluated allelic gene expression. Only $6 / 352$ genes known to be subject to XCI showed $>2$-fold upregulation in $\Delta F i r r e^{\mathrm{Xa}}$, suggesting minor $\mathrm{Xi}$ reactivation (Fig. 6c, Supplementary Data 3 and 7). Four of the reactivated genes were located at the telomeric end of the $\mathrm{Xi}$ where higher accessibility and decreased contact density were observed by ATAC-seq and Hi-C (see below). Although their number is small and we cannot rule out a chance occurrence, more genes known to escape XCI were dysregulated (10-14\%) from the Xi than genes subject to XCI (2-3\%) (Fig. 6c, Supplementary Fig. 4d, Supplementary Data 7). A majority of dysregulated X-linked genes $(67 \%)$ were rescued in $\Delta$ Firre $\mathrm{Xa}+$ mtransgene (Supplementary Fig. 4e, Supplementary Data 3 and 7).

Metagene plots of H3K27me3 along autosomal and X-linked genes grouped based on their expression changes in $\Delta F i r r e^{\mathrm{Xa}}$, together with profiles of individual genes showed a small increase in $\mathrm{H} 3 \mathrm{~K} 27 \mathrm{me} 3$ for downregulated genes, and very little or no change for upregulated or unchanged genes (Supplementary Fig. 5a, b). Note that $90 \%$ of dysregulated genes have no H3K27me3 enrichment in either WT or $\Delta$ Firre $^{\mathrm{Xa}}$. Additional CUT\&RUN analyses of two active epigenetic marks, H3K36me3 and H3K4me3, showed no significant changes on the $\mathrm{Xi}$ in $\Delta$ Firre $^{\mathrm{Xa}}$, consistent with little Xi reactivation (Supplementary Fig. $5 c, d$ )

Thus, the loss of Firre RNA causes widespread changes in gene expression in large part rescued by a cDNA transgene. Very little reactivation and few changes in escape genes occur on the $\mathrm{Xi}$ despite the observed loss of $\mathrm{H} 3 \mathrm{~K} 27 \mathrm{me} 3$, consistent with this epigenetic mark representing only one layer of XCI control ${ }^{20}$.

Allelic alterations of the Firre locus change Xi structure. We evaluated chromatin accessibility in Firre mutants by ATAC-seq. As expected, in WT the distribution of allelic proportions centered at 0.5 for autosomes, but was skewed towards the $\mathrm{Xa}(0.95)$ for the $\mathrm{X}$ chromosomes, indicative of lower chromatin accessibility on the Xi. This pattern remained similar in $\Delta$ Firre ${ }^{\mathrm{Xa}}$, consistent with near absence of gene reactivation. Plots of ATAC peak density further captured the low accessibility profiles of the $\mathrm{Xi}$ in both WT and $\Delta$ Firre $^{\mathrm{Xa}}$ (Fig. 7a, b). However, a higher peak density was observed in the telomeric region of the $\mathrm{Xi}$ in $\Delta$ Firre $^{\mathrm{Xa}}$ where reactivated genes are located (Fig. 6c; 7c). ATAC-seq patterns were unchanged in $\Delta F i r r e^{\mathrm{Xi}}$ and InvFirre $\mathrm{Xi}^{\mathrm{Xi}}$ (Supplementary Fig. 6a, b). To determine whether Dxz4 and Firre may have synergistic cis-effects on chromatin accessibility on the $\mathrm{Xi}$, ATAC-seq was done on a double-mutant line $\Delta$ Firre ${ }^{\mathrm{Xi}} / \Delta D x z 4^{\mathrm{Xi}}$. Interestingly, a pronounced shift to lower values in the distribution of allelic proportions for the $\mathrm{X}$ chromosomes (peak at $\sim 0.55$ ) was observed in this double-mutant compared to either $\Delta D x z 4^{\mathrm{Xi}}$ (peak at $\sim 0.85$ ) or $\Delta$ Firre $^{\mathrm{Xi}}$ (peak at $\sim 1$ ), indicating increased chromatin accessibility on the Xi (Fig. 7d, e).

Based on high-resolution allele-specific contact maps generated by DNase Hi-C the Xi bipartite structure was retained in $\Delta$ Firre ${ }^{\mathrm{Xi}}$, but contacts increased between the superdomains and decreased 
a

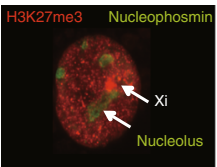

WT

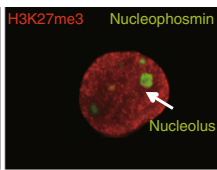

$\Delta$ Firre $^{X_{a}}$

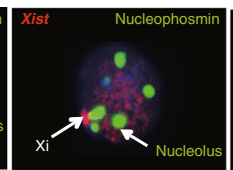

$\Delta$ Firre $^{x_{a}}$

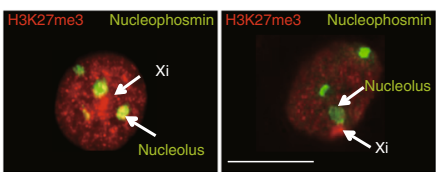

$\Delta$ Firre $^{x_{i}}$

Inv-Firre ${ }^{X_{i}}$

b
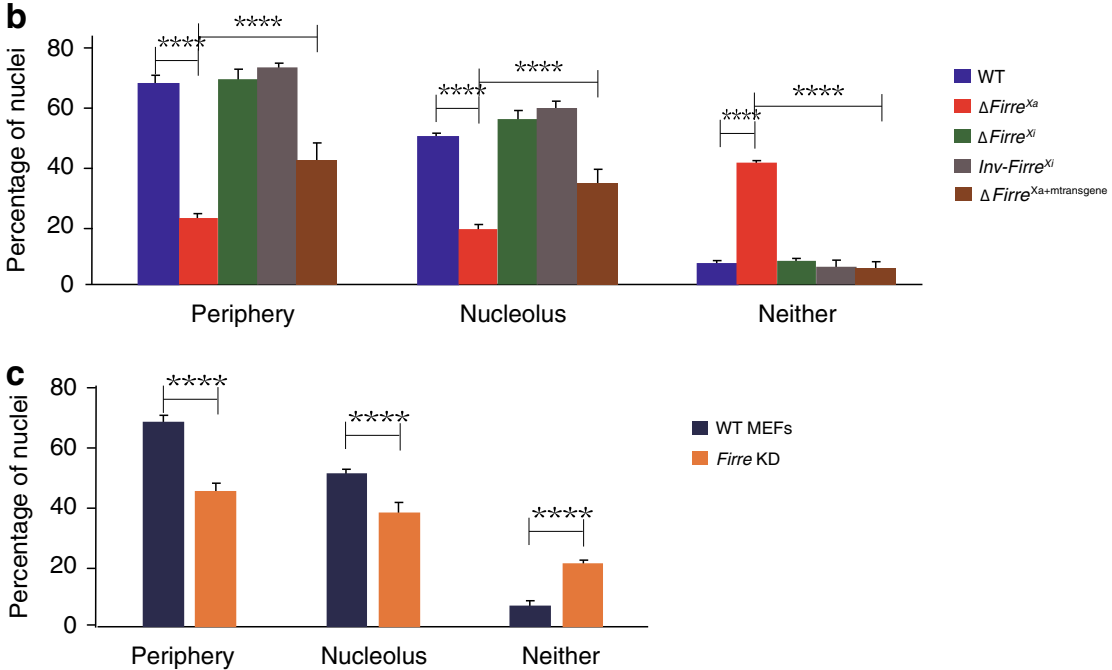

d

CTCF allelic proportions
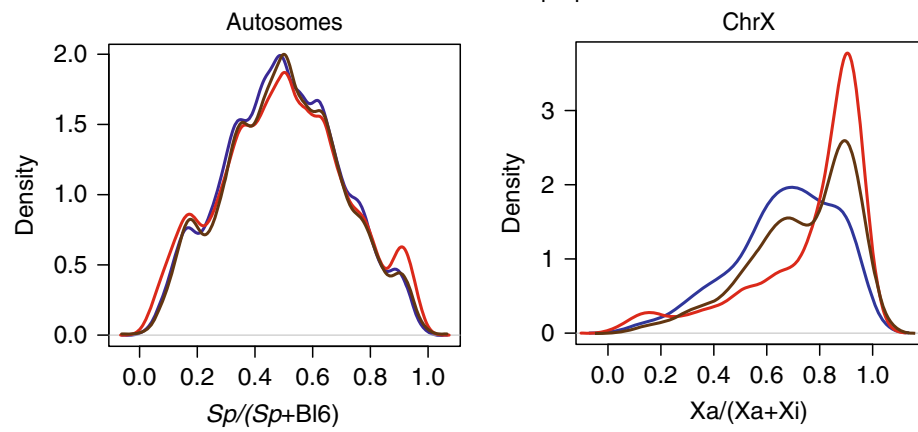

- WT

$\Delta$ Firre $^{X a}$

$\Delta$ Firre $^{\mathrm{X}+\mathrm{a}}$

Firre KD

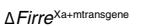

e

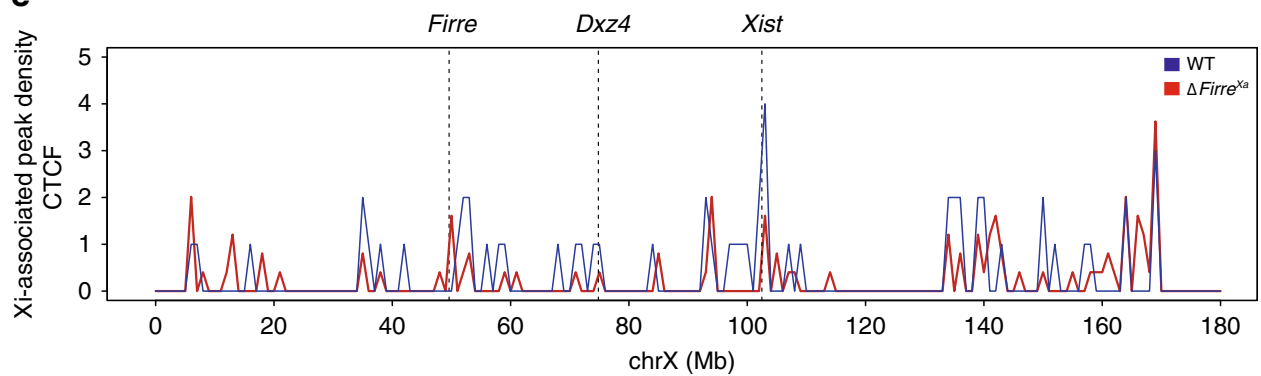

within each superdomain, suggesting that the Firre locus acts in cis to help shape the 3D structure (Fig. 8a). Contacts within regions flanked by Dxz4 and Xist (ChrX:75-100 Mb) and to a lesser extent, flanked by Firre and Dxz4 (ChrX:50-75 Mb) were increased, suggesting disruption of contacts emanating from $D x z 4$ (Fig. 8a, Supplementary Fig. 7a). Similarly, InvFirre $\mathrm{Xi}$ nuclei showed persistence of the $\mathrm{Xi}$ bipartite structure, but also a redistribution of contacts around Firre, including loss of proximal contacts (ChrX:5-50 Mb) and gain of distal contacts (ChrX:50-75 Mb), which was confirmed by virtual $4 \mathrm{C}$ (Fig. 8a, b; Supplementary Fig. 7a). The boundary at or near the Firre locus on the WT Xi was maintained upon deletion or inversion of the locus (Supplementary Fig. 7b). While the loss of Firre RNA in
$\Delta$ Firre $^{\mathrm{Xa}}$ did not perturb the bipartite structure of the $\mathrm{Xi}$, there were some changes in contact distribution, suggesting transeffects (Fig. 8c, Supplementary Fig. 7c). Specifically, contacts increased in the region flanked by Dxz4 and Xist (ChrX:75-100 $\mathrm{Mb})$, and diminished in the very distal telomeric region (ChrX:165-170 Mb), consistent with an increase in chromatin accessibility and gene expression (Figs. 6c, 7c, 8c). Deletion of Firre on the $\mathrm{Xa}$ also resulted in contact changes on the $\mathrm{Xa}$, including loss of the boundary at or close to the locus, as confirmed by insulation score analysis (Fig. 8d; Supplementary Fig. 7d, e).

Taken together, our results indicate cooperation between Firre and Dxz4 in repression of chromatin accessibility on the $\mathrm{Xi}$, with 
Fig. 5 Firre RNA acts in trans to maintain Xi location. a-c A total of $>300$ nuclei per cell type over 3 independent experiments were scored for the location of the Xi marked by a H3K27me3 cluster or an Xist cloud relative to the nuclear periphery or the nucleolus; significance was determined by one-sided Fisher exact test; bar plots are presented as mean values \pm SEM; scale bars represent $10 \mu \mathrm{m}$. a Examples of nuclei after H3K27me3 immunostaining (red) to locate

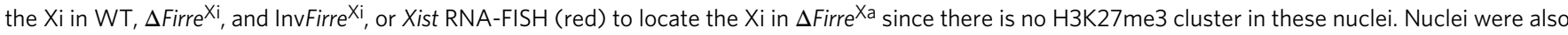
immunostained for NPM1 (green) to locate the nucleolus. b Bar plots show a significant decrease in periphery- and nucleolus association of the Xi in $\Delta$ Firre $^{\mathrm{Xa}}$ compared to WT ( $p$ value $=3.95632 \mathrm{e}-40$ and 3.75497e-14, respectively), but no significant changes in $\Delta$ Firre $^{\mathrm{Xi}}$ and InvFirre ${ }^{X i}(p$ value $=0.6968)$. Ectopic expression of a mouse transgene in $\Delta$ Firre Xa+mtransgene partly rescues the Xi location to $41 \%$ at the periphery and $34 \%$ at the nucleolus $(p$ value $=$ 6.62701e-10 and 0.000265481, respectively). c Bar plots show a significant decrease in periphery- and nucleolus association of the Xi after Firre KD in primary MEFs derived from an F1 embryo (BL6 $\times$ spretus) ( $p$ value $=1.13757 \mathrm{e}-09$ and 0.0000634923 , respectively). d Density histograms of the distribution of allelic proportions $(\mathrm{Xa} /(\mathrm{Xa}+\mathrm{Xi})$ ) of CTCF peaks show a shift in the distribution for the $\mathrm{X}$ chromosomes due to a decrease in CTCF on the $\mathrm{Xi}$

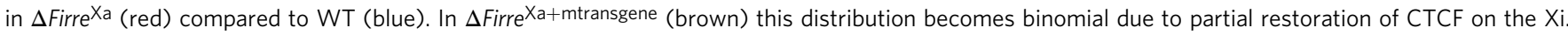
e Plots of Xi-associated (common +Xi-specific) CTCF peak density (counts binned within $100 \mathrm{~kb}$ windows) along the Xi for WT (blue) and $\Delta$ Firre ${ }^{\mathrm{Xa}}$ (red). To account for differences in the number of SNP-covered peaks due to sequencing depth, the binned counts are scaled by a factor obtained from the between-sample ratios of autosomal diploid SNP-covered peaks.

each locus contributing to the two superdomains separation. Firre contacts with other regions on the $\mathrm{Xi}$ appear orientationdependent, reminiscent to the orientation-dependent contacts made by $D x z 4^{29}$. Firre RNA exerts trans-effects on the $\mathrm{Xi} 3 \mathrm{D}$ structure, potentially secondary to losses of $\mathrm{H} 3 \mathrm{~K} 27 \mathrm{me} 3$ and CTCF binding.

\section{Discussion}

Studies of lncRNAs support the notion that these molecules can either spread in cis from their genomic locus or localize to cellular compartments away from their own locus of transcription to perform essential functions in regulating gene expression ${ }^{52-55}$. Here, we report that the lncRNA Firre transcribed from the Xa acts in trans and in cis on the $\mathrm{Xi}$ to maintain its epigenetic features, nuclear location, and 3D structure. LncRNAs have important roles in the structure of nuclei where they fold into higher-order structures and act in cooperation with proteins including chromatin-modifying complexes ${ }^{56}$. Xist represents the quintessential example of a lncRNA that spreads along the $\mathrm{Xi}$ in cis to recruit a series of proteins including components of the PRC complexes that implement chromatin modifications such as $\mathrm{H} 3 \mathrm{~K} 27 \mathrm{me} 3^{2,3,6,19,56}$. We find that maintenance of H3K27me3 on the Xi mediated by Firre RNA involve the PRC complexes, which is supported by our reanalyses of RNA/protein interaction datasets $^{39-45}$. Among Firre interactors EZH2 and SUZ12 represent core subunits of PRC2, while JARID2 and RBFOX2 are cofactors that directly interact with $\mathrm{RNA}^{57-59}$. PRC2 recruitment of the noncanonical PCGF3/5-PRC1 complex is facilitated by the Firre interactor hnRNPK ${ }^{60}$, while $\mathrm{CBX7}$ is a subunit of the canonical PRC1 complex ${ }^{61}$. A new method to detect protein-RNA interactions (incPRINT) also identifies JARID2, EPC1, CTCF, and hnRNPU as Firre interactors ${ }^{62}$. Most Firre interactors have previously been implicated in XCI, and more specifically in $\mathrm{H} 3 \mathrm{~K} 27 \mathrm{me} 3$ enrichment on the Xi (Supplementary Data 5) 2,6,63-66. Colocalization of Firre RNA to the Xi was seen in only $15 \%$ of nuclei, suggesting dynamic binding of the lncRNA. Interestingly, single particle tracking of endogenous EZH2 and SUZ12 in human cells reveals rapid diffusion of PRC2 through the nucleus, with only $\sim 20 \%$ chromatin-bound ${ }^{67}$.

Trans-effects have been reported for several lncRNAs. For example, Fendrr and Pint recruit PRC2 for H3K27 trimethylation of loci located on other chromosomes ${ }^{68,69}$. Meg3 also recruits PRC2 components, JARID2 and EZH2, to facilitate $\mathrm{H} 3 \mathrm{~K} 27 \mathrm{me} 3$ deposition and repression of genes in trans ${ }^{42,70}$. Meg3 has an additional cis-acting role by sequestration of PRC2 to prevent DNA methylation-induced repression of genes within the Meg3-Mirg imprinting cluster ${ }^{71,72}$. Interestingly, we observe a local loss of $\mathrm{H} 3 \mathrm{~K} 27 \mathrm{me} 3$ centering around the Firre locus and extending to $26 \mathrm{Mb}$ in CLPs and CD8 $+\mathrm{T}$ cells from Firre $\mathrm{KO}$ female mice, suggesting that Firre may also act in cis to maintain $\mathrm{H} 3 \mathrm{~K} 27 \mathrm{me} 3$ in certain cell types. Importantly, defects in hematopoiesis that impact CLPs have been observed in Firre KO mice ${ }^{32,33}$. Local cis-effects have also been demonstrated for the imprinted lncRNAs Airn and Kcnq1ot1, which recruit the PRCs via hnRNPK to silence $\mathrm{Mb}$-sized regions on one allele in $\mathrm{cis}^{39}$. Our results based on $\mathrm{KD}$ and transgenic rescue reveal dosedependent effects of Firre RNA on H3K27me3 enrichment on the $\mathrm{Xi}$, reminiscent of the dose-dependent silencing effects of Airn and Kcnqlot $1^{39}$.

The nucleolus has emerged as a platform for the organization of chromatin enriched in repressive histone modifications ${ }^{16-18,73}$. For example, loss of NPM1 results in deformed nucleoli and redistribution of $\mathrm{H} 3 \mathrm{~K} 27 \mathrm{me} 3^{74}$. Depletion of Firre RNA limits association of the $\mathrm{Xi}$ to the nucleolus and nuclear periphery, supporting coordinated roles in $\mathrm{Xi}$ positioning and maintenance of $\mathrm{H} 3 \mathrm{~K} 27 \mathrm{me} 3^{20}$. Two studies corroborate our findings: indeed, relocation of the $\mathrm{Xi}$ to the nucleolus during the cell cycle is required to maintain $\mathrm{H} 3 \mathrm{~K} 27 \mathrm{me} 3$, and sparser $\mathrm{H} 3 \mathrm{~K} 27 \mathrm{me} 3$ on the $\mathrm{Xi}$ occurs when it is kept away from the nucleolus or nuclear periphery ${ }^{75,76}$. Deletion of Xist also decreases both H3K27me3 enrichment and nucleolar association of the $\mathrm{Xi}^{77}$. However, we did not observe disruption of Xist RNA expression nor coating of the $\mathrm{Xi}$ in $\Delta$ Firre $^{\mathrm{Xa}}$ nuclei, suggesting that Firre acts independently of Xist. On the other hand, CTCF, a protein that facilitates interactions between chromatin and the nucleolus may act in concert with Firre to help Xi positioning ${ }^{51}$. In addition to CTCFDNA interactions CTCF-RNA interactions via specific zinc fingers represent important structural components of genome organization $^{78}$. Such dual interactions characterize Firre since CTCF not only binds to the locus on the $\mathrm{Xi}$, but also interacts with the RNA $20,21,62$. Thus, in the absence of Firre RNA disrupted $\mathrm{Xi}$ anchoring to the nucleolar periphery could result from the significant loss of CTCF binding observed along the entire $\mathrm{Xi}$, including at the Firre locus. Supporting this notion, RNA depletion using RNase A disrupts the local chromatin environment around CTCF binding sites and the structural integrity of heterochromatin ${ }^{38}$. We cannot exclude other heterochromatin factors, for example, EZH1 or histone H1, both downregulated in $\Delta$ Firre $^{\mathrm{Xa}}$ and rescued in transgenic lines ${ }^{79,80}$. Considering the $3 \mathrm{D}$ structure of the $\mathrm{Xi}$, the Firre locus helps insulate the two superdomains of the Xi perhaps via CTCF binding and acts in synergy with $D x z 4$ to compact the $\mathrm{Xi}$ perhaps via a superloop with $D x z 4^{27}$

Our rescue experiments imply that a subset of Firre exons are sufficient for partial maintenance of $\mathrm{H} 3 \mathrm{~K} 27 \mathrm{me} 3$ on the $\mathrm{Xi}$ and its location in the nucleus. It will be interesting to map additional functional units within the Firre locus, which may provide a more complete rescue. The Firre locus harbors several conserved local 
a

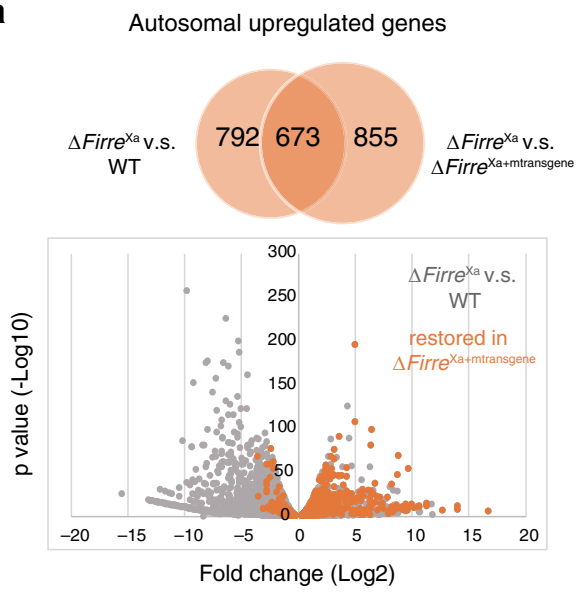

b
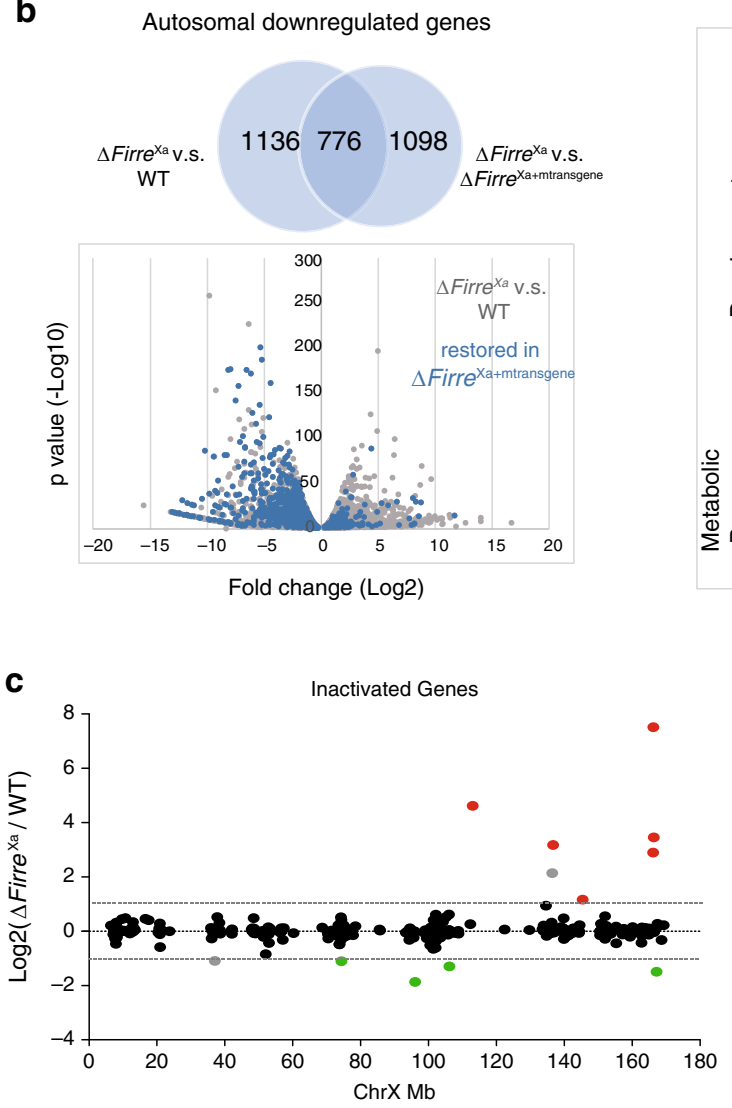
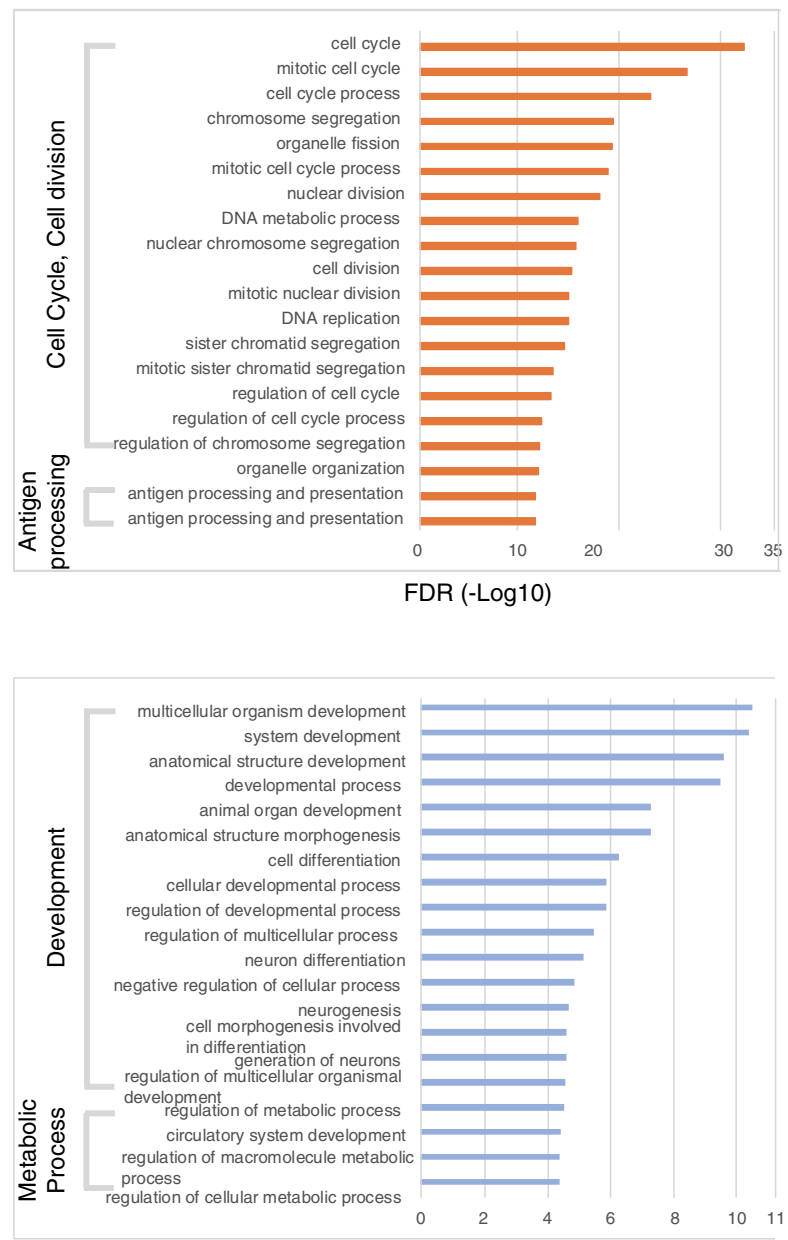

FDR (-Log10)

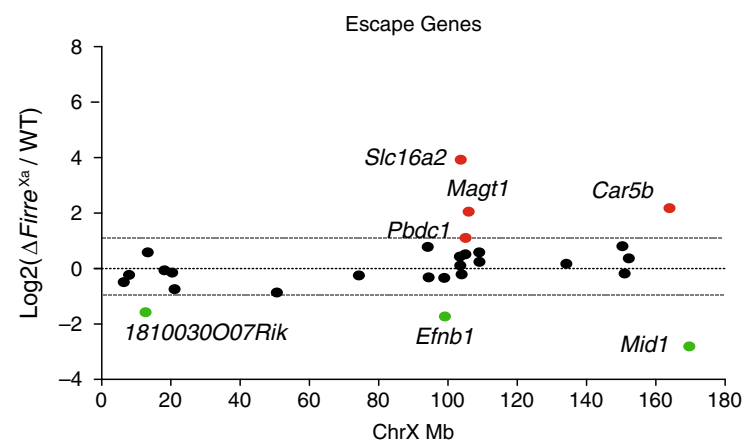

Fig. 6 Loss of Firre RNA affects gene expression. a Upregulated genes in $\Delta$ Firre ${ }^{X a}$ and Gene Ontology (GO) term enrichment. The Venn diagram shows the number of upregulated genes in $\Delta$ Firre ${ }^{\mathrm{X} a}$ versus WT, and in $\Delta$ Firre $^{\mathrm{Xa}}$ versus $\Delta$ Firre $\mathrm{Xa}$-mtransgene, with the overlapping gene set representing upregulated genes in $\Delta$ Firre ${ }^{X a}$ that are rescued by transgene expression. The scatter plot shows dysregulated genes in $\Delta$ Firre ${ }^{X a}$ versus WT (grey), with genes rescued by reduced expression in $\Delta$ Firre ${ }^{\mathrm{Xa}+\text { mtransgene }}$ versus $\Delta$ Firre ${ }^{\mathrm{X} a}$ (more than 2 -fold; $p$ value $<0.05$ by the Wald test) highlighted in orange. The top $20 \mathrm{GO}$ terms of overlapping upregulated genes in $\Delta$ Firre ${ }^{X}$ a versus WT, which are rescued in $\Delta$ Firre ${ }^{X a+m t r a n s g e n e}$ are listed. The $X$-axis indicates the FDR ( - log10). b Downregulated genes in $\Delta$ Firre ${ }^{X a}$ and Gene Ontology (GO) term enrichment. The Venn diagram shows the number of downregulated genes in $\Delta F i r r X^{X}$

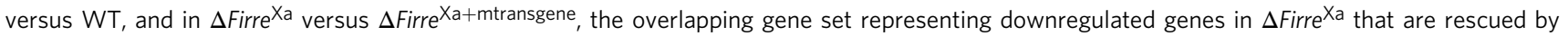
transgene expression. The scatter plot shows dysregulated genes in $\Delta$ Firre ${ }^{X a}$ versus WT (gray), with genes rescued by increased expression in $\Delta$ Firre ${ }^{\mathrm{Xa}+\text { mtransgene }}$ versus $\Delta$ Firre ${ }^{\mathrm{Xa}}$ (more than 2 -fold; $p$ value $<0.05$ by the Wald test) highlighted in blue. The top $20 \mathrm{GO}$ terms of overlapping downregulated genes in $\Delta$ Firre ${ }^{\mathrm{Xa}}$ versus WT, which are rescued in $\Delta$ Firre $\mathrm{Xa}_{\mathrm{a}}$ mtransgene are listed. The $\mathrm{X}$-axis indicates the FDR (-log10). c Xi-expression fold changes for genes that are subject to or escape $\mathrm{XCl}$ between $\Delta$ Firre $\mathrm{Xa}^{\mathrm{a}}$ and $\mathrm{WT}$. Upregulated genes are in red and downregulated genes in green. To note, one upregulated gene and one gene subject to $\mathrm{XCl}$ are in gray, as they showed more than 2-fold change in expression but with a $p$ value $>0.05$ by the Wald test in $\triangle$ Firre $^{\mathrm{Xa}}$ versus WT. Genes are ordered from centromere to telomere along the Xi. 
a

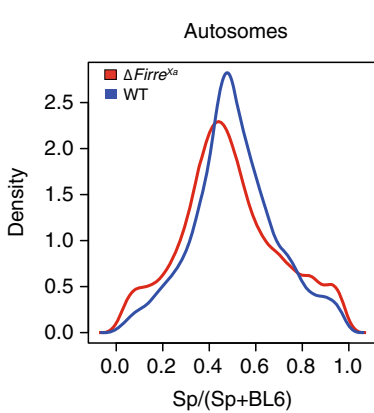

ATAC allelic proportions

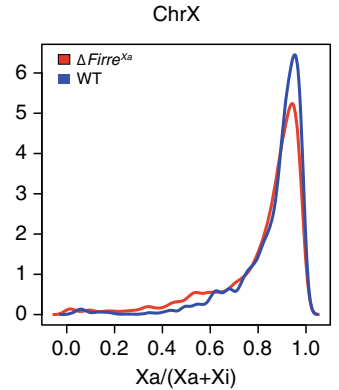

b

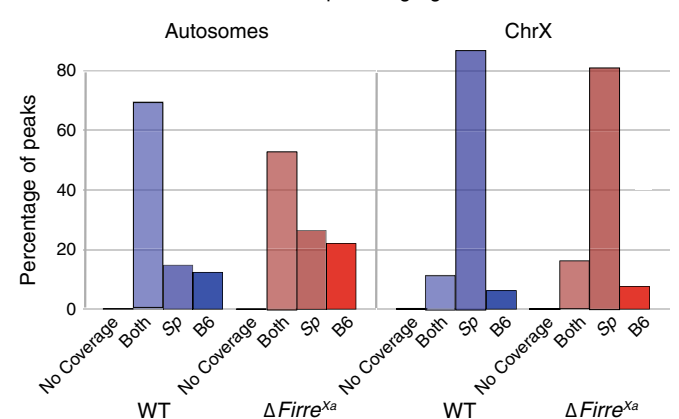

C

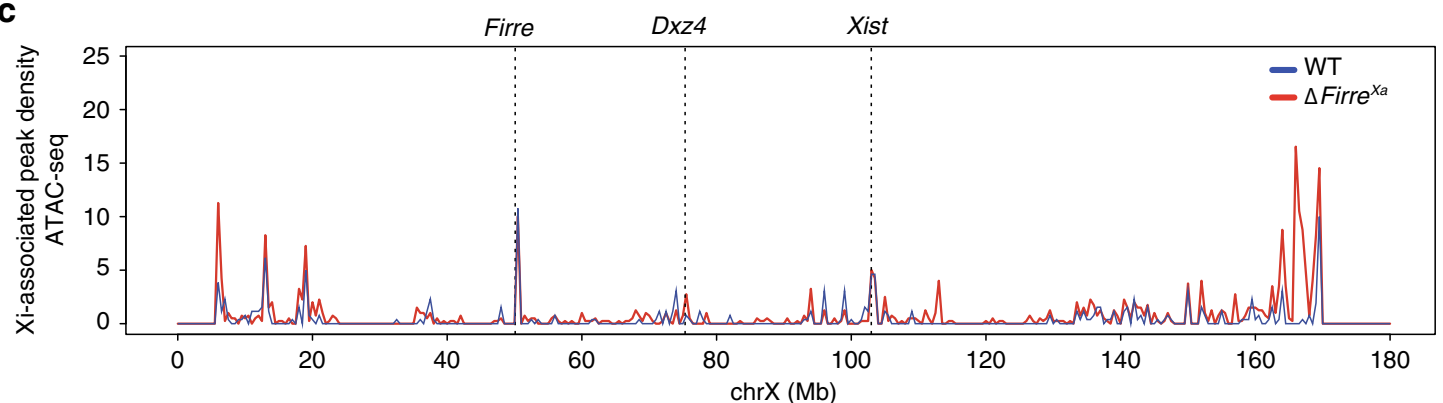

d

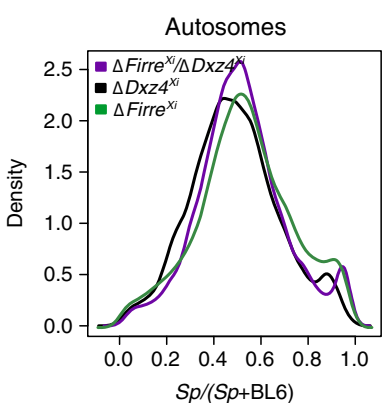

ATAC allelic proportions

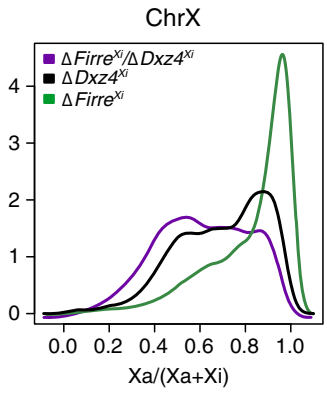

e

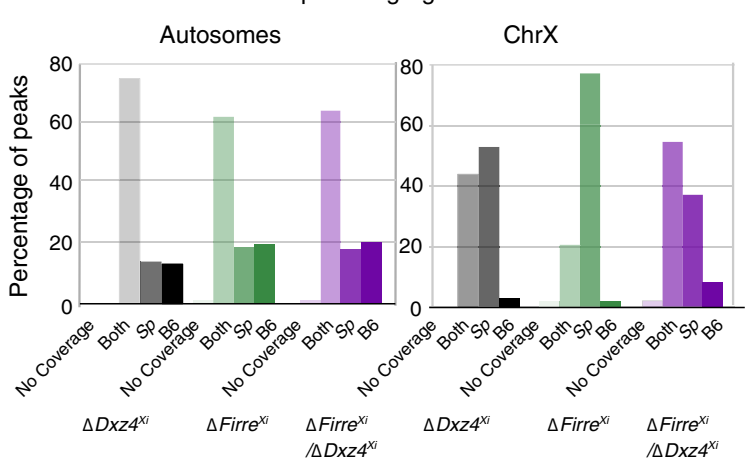

Fig. 7 Chromatin accessibility after allelic Firre deletions and a Firre/Dxz4 deletion. a Density histograms of the distribution of allelic proportions (spretus/(spretus + BL6)) of ATAC peaks along the autosomes and the X chromosomes for WT (blue) and $\Delta$ Firre ${ }^{X a}$ (red). No shift is observed (Wilcoxon test: $-\log 10 P=32$ ). b. Percentages of ATAC peaks along the autosomes and the $X$ chromosomes classified as spretus-specific, BL6-specific, or both show no differences between WT (blue) and $\Delta$ Firre ${ }^{X a}$ (red). c. Plots of Xi-associated (common + Xi-specific) ATAC peak density (counts binned within 500 kb

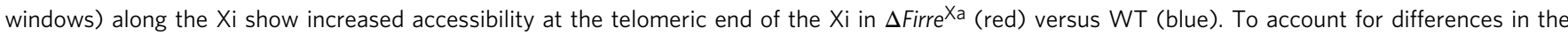
number of SNP-covered peaks between samples due to sequencing depth, the binned counts are scaled by a factor obtained from the between-sample ratios of autosomal diploid SNP-covered peaks. d Density histograms of the distribution of allelic proportions (spretus/(spretus + BL6)) of ATAC peaks show a shift to a lower $\mathrm{Xa} /(\mathrm{Xa}+\mathrm{Xi})$ ratio in the double-mutant $\Delta$ Firre $\mathrm{Xi}_{\mathrm{i}} \Delta D x z 4^{\mathrm{Xi}}$ (purple), compared to $\Delta D x z 4^{\mathrm{X} i}$ (black) and $\Delta$ Firre $\mathrm{Xi}^{\mathrm{i}}$ (green), consistent with increased accessibility on the Xi (Wilcoxon test: $-\log 10 P=35$ ). e Percentages of ATAC peaks in $\Delta D x z 4^{X i}$ (black), $\Delta$ Firre ${ }^{X i}$ (green), and $\Delta$ Firre ${ }^{X i}$ / $\Delta D x z 4^{X i}$ (purple) along the autosomes and the $X$ chromosomes classified as spretus-specific, BL6-specific, or both show an increase on the BL6 $X i$ in the double mutant.

repeats and many of these do not overlap exons, except for the RRD repeats that display $65 \%$ identity between human and mouse, which makes them good candidates for the rescue function observed ${ }^{21,23}$. Notably, these repeats bind the nuclear matrix protein hnRNPU known to associate with Xist, the $\mathrm{Xi}$, and other genomic regions ${ }^{20,21,24,81-84}$.

Our findings of dysregulated genes implicated in cell cycle and development upon loss of Firre RNA support a role in cell growth, a common finding for other lncRNAs ${ }^{85}$. Both Firre KO mice and mice with Firre overexpression have abnormalities specifically in hematopoiesis, implying the importance of Firre dosage $^{32,33}$. In human loss of FIRRE RNA causes dysregulation of inflammatory gene expression, while amplification of FIRRE causes congenital abnormalities and is associated with decreased survival rates in cancer, further supporting FIRRE dosage effects $^{24,85,86}$.

Despite ample evidence supporting their biological relevance in cell systems lncRNAs are often dispensable for survival of vertebrate organisms. For example, mice homozygous for deletions of regions harboring 1243 noncoding sequences had no distinguishable phenotypes ${ }^{87}$. Similarly, mice with deletions of nine conserved lncRNAs including Malat were viable with no obvious abnormalities ${ }^{50,88}$. Firre KO mice have few abnormal phenotypes except for abnormalities in B- and T-cell physiology $^{33}$. This dearth of phenotypes could be explained by alternative pathways, possibly other lncRNAs that may compensate 
a

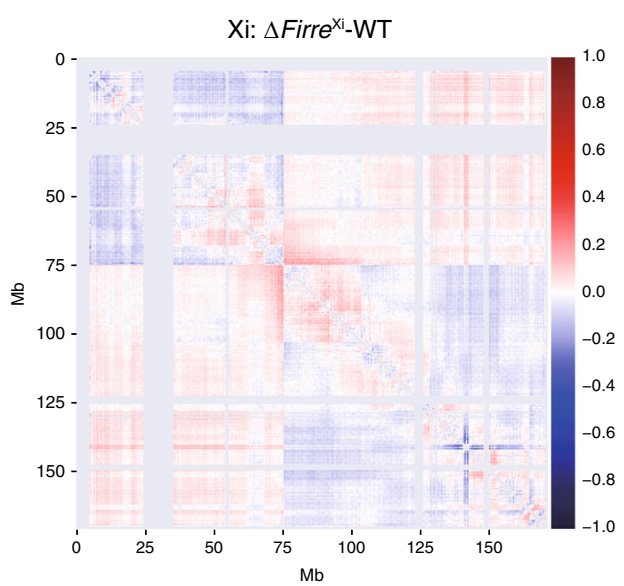

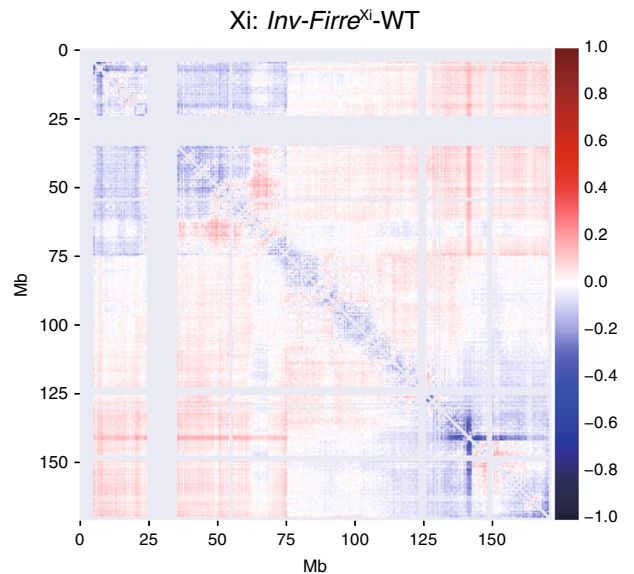

b 100
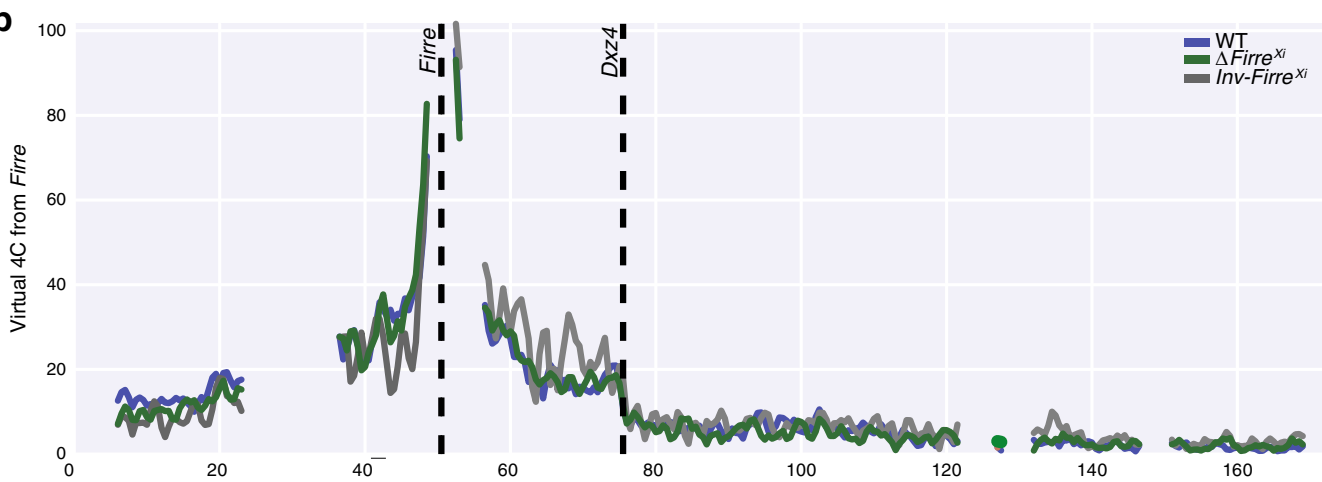

C

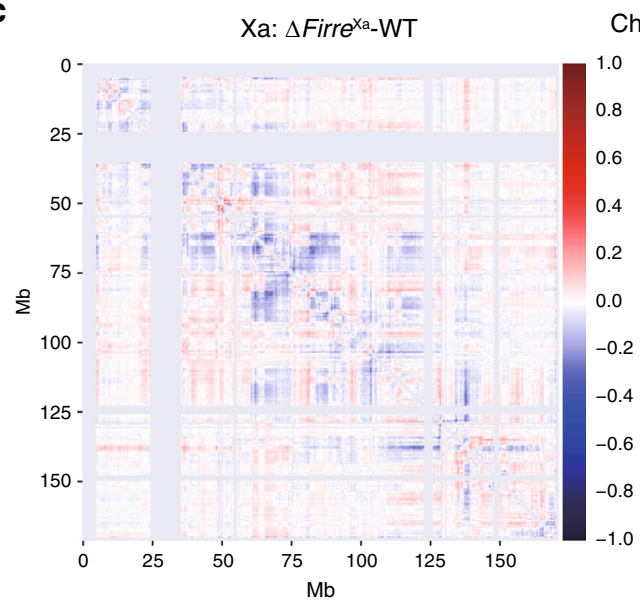

$\mathrm{ChrX} \mathrm{Mb}$

d

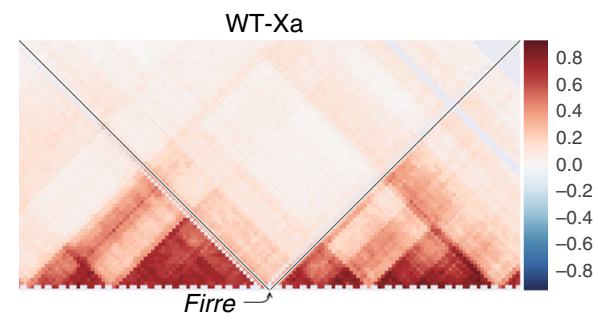

during development, providing redundancy in the system ${ }^{89}$. A recent publication reports that following 10 days of differentiation, mouse ES cells with a Firre deletion on the Xa, the Xi or on both alleles show no effects on $\mathrm{H} 3 \mathrm{~K} 27 \mathrm{me} 3$ on the $\mathrm{Xi}$, which differs from our results based on mutations induced in differentiated fibroblasts ${ }^{35}$. Thus, phenotypic effects apparently depend on the developmental timing of Firre mutations, and mutations at
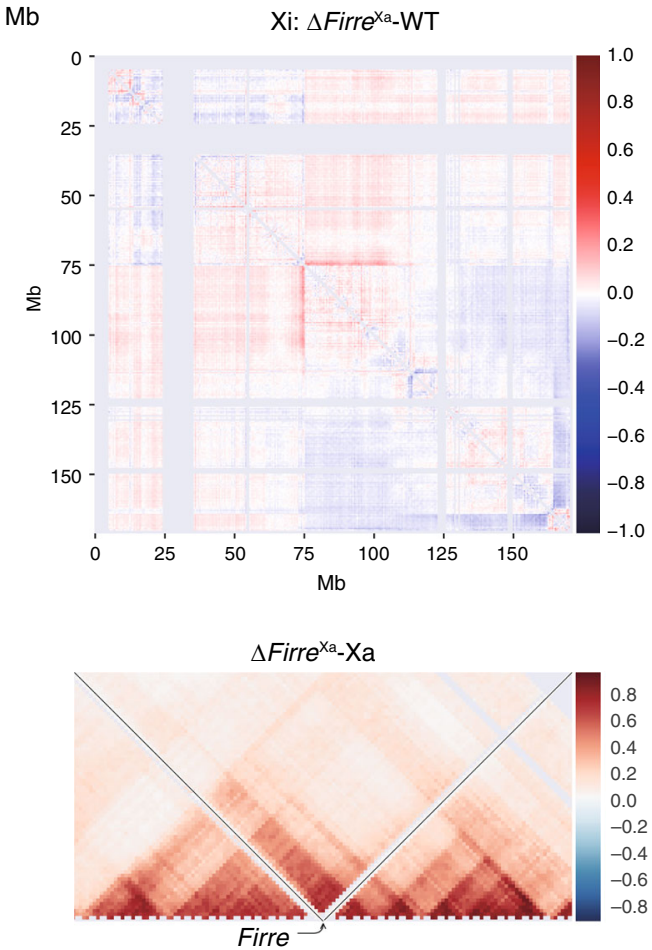

early stages may allow compensatory pathways to effectively help survival. Indeed, Firre KO ES cells display a marked decrease in growth rate, supporting the existence of critical mechanisms for survival of subsets of cells and subsequent differentiation/development ${ }^{23}$. In addition, differences in the effects of Firre mutations may reflect developmental stage-specific composition of PRC complexes ${ }^{90}$. 
Fig. 8 Changes in the $\mathbf{X}$ 3D structure after Firre deletion or inversion. a Pearson correlated-transformed differential contact maps of the $X i$ at $500 \mathrm{~kb}$ resolution highlight differences between $\Delta F_{i r r e}{ }^{X}$ and $W T$, and between InvFirre ${ }^{X i}$ and WT. The color scale shows differential Pearson correlation values, with loss and gain of contacts in the mutants versus WT appearing blue and red, respectively. See text for description of changes. $\mathbf{b}$ Virtual $4 \mathrm{C}$ plots

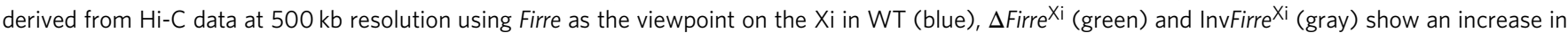
contacts between Firre and Dxz4 in InvFirre ${ }^{X i}$. c Pearson correlated-transformed differential contact maps of the $X a$ and $X i$ at 500 kb resolution to highlight differences between $\Delta$ Firre ${ }^{X a}$ and WT. The color scale shows differential Pearson correlation values, with loss and gain of contacts in $\Delta F i r r e{ }^{X a}$ versus WT appearing blue and red, respectively. See text for description of changes. d Pearson correlated-transformed contact maps ( $40 \mathrm{~kb}$ resolution) of the Xa for 4 $\mathrm{Mb}$ around the Firre locus highlight the loss of the strong boundary between TADs on the Xa in $\Delta$ Firre ${ }^{\mathrm{Xa}}$ versus WT (see Supplementary Fig. 7c for corresponding maps of the $\mathrm{Xi}$ ).

Another important consideration is cell type specificity. There was no apparent loss of $\mathrm{H} 3 \mathrm{~K} 27 \mathrm{me} 3$ on the $\mathrm{Xi}$ in brain, kidney, and liver from Firre KO mice. However, a local disruption of H3K27me3 around Firre was found in CLPs and CD8 + T cells, suggesting that Firre RNA may act in cis in certain cell types. In a CRISPRi phenotypic screen a majority of lncRNAs only displayed phenotypes in a single cell type ${ }^{50}$. A new approach, PIRCh-seq, designed to find association between RNA and specific chromatin modifications reports Firre interactions with H3K27me3 chromatin in MEFs, but not in ES cells, highlighting differences between cell types ${ }^{91}$. Furthermore, mouse and human Firre/FIRRE exhibit diverse expression patterns across tissues, e.g., enrichment in neural crest but depletion in lung fibroblasts ${ }^{23}$. Firre KO mice show organ-dependent gene dysregulation, with a larger number of dysregulated genes in spleen, which can be rescued by ectopic Firre expression supporting a trans-acting role in vivo ${ }^{32,33}$. The gene expression changes we observed in kidneyderived Patski cells differ from those reported in Firre KO mouse spleen, again suggesting tissue-specificity. In immune cells where Firre RNA exerts a local cis-effect on the $\mathrm{Xi}$, it remains to be determined whether Firre might be expressed from the Xi. Interestingly, female lymphocytes lack Xist clouds and $\mathrm{H} 3 \mathrm{~K} 27 \mathrm{me} 3$ foci, and show reactivation of the $\mathrm{Xi}^{92}$. Further analyses to distinguish alleles is needed to better understand Firre local effects in immune cells.

\begin{abstract}
Methods
Cell lines. The Patski cell line, originally derived from embryonic kidney (18.5 dpc) from a cross between a female C57BL/6 J (BL6) with an $\mathrm{Hprt}^{B M 3}$ mutation and a male Mus spretus (spretus), was previously selected in HAT (hypoxanthine-aminopterin-thymidine) medium so that the $\mathrm{Xi}$ is always from BL6 ${ }^{93,94}$. Primary MEF cultures were derived from a $13.5 \mathrm{dpc}$ female embryo resulting from a BL6 $\times$ Mus spretus cross, which results in skewed inactivation of the spretus $\mathrm{X}$ chromosome due to an Xist mutation on the BL6 X chromosome ${ }^{95}$. Primary MEFs were also derived from BL6 $\times$ Mus spretus and BL6 $\times$ Mus castaneus $13.5 \mathrm{dpc}$ female F1 embryos with random XCI. Cells were cultured as described and the presence of normal X chromosomes verified by karyotyping ${ }^{29}$.

For ectopic Firre expression assays in Patski cells and in MEFs, a mouse Firre cDNA plasmid (mtransgene; Dharmacon BC055934) or a human FIRRE cDNA plasmid (htransgene; Dharmacon BC038558) were each transfected together with the selectable marker pPGK-Puro plasmid (gift from R. Jaenisch; Addgene 11349) into $\Delta$ Firre $^{\mathrm{Xa}}$ cells using lipofectamine 3000 (Invitrogen). Blast searches were performed to map homology of the cDNAs to the reference genomes (Supplementary Fig. 1b). After transfection, $\Delta$ Firre $^{\mathrm{Xa}+\text { mtransgene }}$ and $\Delta$ Firre $^{\mathrm{Xa}}$ +htransgene were selected in Eagle's medium with $2 \mu \mathrm{g} / \mathrm{ml}$ puromycin for $72 \mathrm{~h}$, followed by recovery in Eagle's medium with $1 \mu \mathrm{g} / \mathrm{ml}$ puromycin for 10 days. A $\Delta$ Firre $^{\mathrm{Xa}+\text { mtransgene }}$ clone with high Firre expression was also isolated.
\end{abstract}

KO mouse tissues and isolation of CLPs and CD8 $+\mathbf{T}$ cells. Mice used in this study were maintained in pathogen-specific-free facilities under the supervision of either the University of Washington Institutional Animal Care and Use Committee (Protocol number 2254) or Harvard University's Institutional Animal Care Committee. We complied with the ethical regulations for animal testing and research in accordance with these committees. Tissues collected from a Firre KO mouse model included liver, kidney, and brain from heterozygous $\left(\right.$ Firre $\left.^{+/-}\right)$and homozygous $\left(\right.$ Firre $\left.^{-/-}\right)$mutants, and from control female mice verified by genotyping ${ }^{33}$. Ectopic expression of Firre induced by doxycycline (DOX) injection in mice was done as described ${ }^{33}$. MEFs were derived from mutant Firre $^{+/-}$, Firre $^{-1-}$ ) and control female $13.5 \mathrm{dpc}$ embryos.
We isolated CLPs live [Lin-Sca-1locKitloIL7R $a+$ ] by fluorescence-activated cell sorting (FACS) from WT (age: 35-43 days) and Firre ${ }^{-1-}$ (age: 35 days) female mice to generate two replicate samples per genotype, consisting of two mice per replicate. Bone marrow was isolated from both femurs and tibias of each mouse by removing the end caps and flushing the bone marrow with a $27 \mathrm{G}$ syringe containing staining media (DMEM, Gibco, 11995-073) with 5\% fetal bovine serum (FBS, Gibco, 26140079) and $10 \mathrm{mM}$ EDTA into a $50 \mathrm{~mL}$ conical tube. Cells pelleted by centrifugation at $1200 \mathrm{rpm}$ were subjected to lineage depletion according to the manufacturer's protocol (Miltenyi Biotec, 130-090-858). Lineage-depleted bone marrow was resuspended with the following antibodies (1:100): PE/Cy7 anti-mouse CD127 (IL-7Ra) clone A7R34 (Biolegend, 135014), Alexa Fluor 488 anti-mouse CD117 (c-Kit) clone 2B8 (Biolegend, 105816), PE/Dazzle-594 anti-mouse Ly-6A/E (Sca-1) clone D7 (Biolegend, 108138), APC anti-mouse CD34 clone HM34 (Biolegend, 128612), PE anti-mouse CD135 clone A2F10 (Biolegend, 135306), and Pacific Blue anti-mouse Lineage Cocktail $(20 \mu \mathrm{L}$ per $1 \times 106$ cells $)$ clones $17 \mathrm{~A} 2 /$ RB6-8C5/RA3-6B2/Ter-119/M1/70 (Biolegend, 133310). Zombie Aqua Fixable Viability Kit (Biolegend, 423101) was used as a viability stain according to the manufacturer's protocol. Samples were incubated on ice in the dark for $60 \mathrm{~min}$, washed twice with staining media, and resuspended before sorting by FACS (BD Aria).

To isolate CD8 + T-cells peripheral blood was collected from mice by cardiac puncture into a tube containing $4 \%$ citrate. Red blood cells were lysed for $15 \mathrm{~min}$ a room temperature using BD Pharm Lyse (BD, 555899). Cells were washed twice with staining media and the following antibodies were added (1:100) to each sample prior to incubation for $30 \mathrm{~min}$ at room temperature: $\mathrm{PE}$ anti-mouse CD3 clone 17A2 (Biolegend, 100205), Alexa Fluor 700 anti-mouse CD8a clone 53-6.7 (Biolegend, 100730), APC anti-mouse CD19 clone 6D5 (Biolegend, 115512), Alexa Fluor 488 anti-mouse NK-1.1 clone PK136 (Biolegend, 108718), PE/Dazzle-594 anti-mouse CD4 clone GK1.5 (Biolegend, 100456), TruStain FcX (anti-mouse CD16/32) clone 93 (1:50) (Biolegend, 101319). Zombie Aqua Fixable Viability Kit (Biolegend, 423101) was used as a viability stain. Cells were washed twice with staining media and sorted by FACS (BD Aria).

Allele-specific CRISPR/Cas9 editing and RNAi KD. For allele-specific CRISPR/ Cas9 editing of the endogenous Firre locus, three highly specific sgRNAs with BL6 or spretus SNPs at the PAM site and with low off-target scores were chosen and aligned back to the reference genome using BLAT (UCSC) to verify specificity (Supplementary Data 1 ). The sgRNAs cloned into $\mathrm{p} \times 330$ plasmids (Addgene) were transfected into WT Patski cells using Ultracruz reagents (Santa Cruz). A Patski line with a deletion of $D x z 4$ was also transfected to generate a double-mutant $\Delta$ Firre $^{\mathrm{Xi}} / \Delta D x z 4^{\mathrm{Xi}} 29$. Single-cell derived colonies were selected and deletions or inversions of the targeted Firre locus verified using PCR and Sanger sequencing to confirm allele-specific editing (Supplementary Data 2). Firre RNAi KD was performed as described ${ }^{20}$. Cells were harvested after double shRNA/siRNA treatment and qRT-PCR performed to verify KD efficiency.

Immunofluorescence, RNA-FISH, and DNA-FISH. Immunofluorescence was done on cells grown on chamber slides, fixed with paraformaldehyde, permeabilized, and blocked as described previously ${ }^{20}$. Mouse liver, kidney, and brain were embedded in a cassette and sectioned by the University of Washington histopathology service center. Tissue sections $(5 \mu \mathrm{m})$ were permeabilized using $0.5 \%$ Triton X-100 for $10 \mathrm{~min}$ and fixed in $4 \%$ paraformaldehyde for $10 \mathrm{~min}$. After incubation with a primary antibody specific for H3K27me3 (Upstate/Millipore, \#07-449), H2AK119ubi (Cell Signaling, \#8240 S), macroH2A (Abcam, \#ab37264), or NPM1 (Abcam, \#ab10530) overnight at $4{ }^{\circ} \mathrm{C}$ in a humidified chamber cells/ tissue sections were washed in $1 \times$ PBS (phosphate-buffered saline) buffer and incubated with a secondary antibody conjugated to Texas Red (anti-rabbit, Vector, \# TI-1000) or fluorescein (anti-mouse, Vector, \# FI-2100). RNA-FISH for Xist was done using a labeled $10 \mathrm{~kb}$ cDNA plasmid (pXho, which contains most of Xist exon 1 ) as described ${ }^{20}$. RNA-FISH for Firre was done in a clonal $\Delta$ Firre $^{\mathrm{Xa}+\text { mtransgene }}$ line that overexpresses Firre RNA using a labeled plasmid probe containing Firre cDNA. DNA-FISH was done using labeled BAC probes containing Firre (RP24$322 \mathrm{~N} 20)$ or Dxz4 (RP23-299L1) ${ }^{29}$. Images were acquired with a Zeiss fluorescence microscope equipped with an image capture system ZEN 2.3. 
Slides were examined by fluorescence microscopy to score the number of nuclei with enrichment in each histone modification on the Xi, using Xist RNA-FISH for $\mathrm{Xi}$ identification. A minimum of 300 nuclei were scored per cell type by at least two observers. Measurements of overall H3K27me3 staining intensity outside the Xi cluster were done in a minimum of 300 nuclei using Image ${ }^{36}$. To minimize experimental variance, a mixture of $80 \% \Delta F$ irre $\mathrm{Xa}^{\mathrm{Xa}}$ and $20 \%$ WT cells were grown in the same chamber prior to immunostaining with $\mathrm{H} 3 \mathrm{~K} 27 \mathrm{me} 3$ and a control histone panH4 (Abcam ab10158), together with DNA counterstaining using Hoechst 33342. Selected nuclear areas away from the Xi were used for measurement of H3K27me3 median intensity, with same-area normalization to either Hoechst 33342 or panH4 staining. Comparisons between WT and $\Delta$ Firre $^{\mathrm{Xa}}$ were done by calculating either the H3K27me3 staining intensity versus Hoechst 33342 and panH4 for WT and $\Delta$ Firre $^{\mathrm{Xa}}$ nuclei separately, or the relative H3K27me3 staining intensity in $\Delta$ Firre $^{\mathrm{Xa}}$ versus WT nuclei present in the same microscope field. The location of the Xi with respect to the nuclear periphery and the edge of the nucleolus (labeled with NPM1) was recorded in at least 300 nuclei.

Allelic in situ Hi-C, CUT\&RUN, ATAC-, RNA-, and ChIP-seq. In situ DNase Hi$\mathrm{C}$ was done on intact nuclei from $\Delta$ Firre $^{\mathrm{Xa}}, \Delta$ Firre $^{\mathrm{Xi}}, \operatorname{Inv}$ Firre $^{\mathrm{Xi}}$, and WT as described ${ }^{29,30}$. Hi-C libraries were sequenced using $150 \mathrm{bp}$ paired-end reads (Supplementary Data 11). ATAC-seq on $\Delta$ Firre $^{\mathrm{Xa}}, \Delta$ Firre $^{\mathrm{Xi}}$, InvFirre ${ }^{\mathrm{Xi}}, \Delta$ Firre $^{\mathrm{Xi}}$ $\Delta D x z 4^{\mathrm{Xi}}$, and WT, and RNA-seq on $\Delta$ Firre $^{\mathrm{Xa}}, \Delta$ Firre $^{\mathrm{Xa}+\mathrm{m} \text { transgene, and WT were }}$ done as previously described ${ }^{29}$. ChIP-seq was done on $\Delta$ Firre $^{\mathrm{Xa}}$ and WT using an antibody for H3K27me3 and an established protocol ${ }^{20}$. CUT\&RUN was done on $\triangle F_{i r r e}{ }^{\mathrm{Xa}}, \Delta$ Firre $^{\mathrm{Xa}+\text { mtransgene, }}$, and WT using an antibody for CTCF (Upstate/ Millipore, 07-729-25UL), and on $\Delta$ Firre ${ }^{\mathrm{Xa}}$ and WT using an antibody for SUZ12 (Abcam ab12073) using a published protocol ${ }^{96}$. H3K27me3 ChIP analyses of CLPs and CD8 $+\mathrm{T}$ cells were done using the TrueMicroChIP kit (Diagenode, C01010130) according to the manufacturer's protocol. Briefly, we sheared fixed chromatin from $\sim 32,000$ CLPs and $\sim 50,000$ CD8 + T cells in separate $1.5 \mathrm{~mL}$ TPX plastic tubes (Diagenode, C3001001050) using the following cycles on a Bioruptor (Diagenode): eight cycles of $5 \mathrm{~min}$ ( $30 \mathrm{ON}, 30 \mathrm{OFF}$ ). Between each cycle samples were placed on ice. Sheared chromatin was then immunoprecipitated using $1 \mu \mathrm{L}$ of H3K27me3 antibody (Diagenode, C15410069), retaining 10\% of the chromatin as input. ChIP products were eluted in $12 \mu \mathrm{L}$ and libraries prepared using the MicroPlex Library Preparation kit v2 (Diagenode, C05010012). Ten microleter of ChIP products were used as input, iPCRtagT1-T12 were used for 12 amplification cycles. After library clean up, the libraries were analyzed on a BioAnalyzer DNAHS chip.

ATAC-seq, ChIP-seq, and CUT\&RUN libraries for Patski cells were sequenced as 75 bp pair-end reads (Supplementary Data 11). RNA-seq libraries for Patski cells were sequenced as $75 \mathrm{bp}$ single-end reads (Supplementary Data 11 ). Sequencing datasets were analyzed to assign reads to the spretus or BL6 genomes using a previously developed allele-specific data analysis pipeline ${ }^{29}$. RNA-seq reads were mapped to the UCSC mm10 (NCBI build v38) refSeq BL6 mouse transcriptome. Tophat2 (v 2.0.12) (calling bowtie2 (v2.2.3)) was used to perform single-end mapping allowing six mismatches. Mapped reads were assigned to refSeq genes using HT-seq(v0.11.0) and counts were converted into TPMs using custom R scripts. DE analysis was performed using DESeq2. ATAC-seq, ChIP-seq, and CUT\&RUN reads were mapped to the BL6 mouse genome using the NCBI build $\mathrm{v} 38 / \mathrm{mm} 10$ reference genome assembly obtained from the UCSC Genome Browser using BWA-MEM (v0.7.3) in paired-end mode using default parameters. Peaks were called using MACS2. DNase Hi-C reads were mapped to the BL6 genome using the NCBI build $\mathrm{v} 38 / \mathrm{mm} 10$ reference genome assembly obtained from the UCSC Genome Browser and a pseudo-spretus genome using BWA-MEM (v0.7.3) in single-end mode using default parameters ${ }^{29}$. Micro-RNA-seq (less than 200nt) was done in $\triangle F i r r e^{\mathrm{Xa}}$ and WT Patski cells by BGI Genomics (https://www.bgi.com/ us/). ChIP-seq libraries for CLPs and CD8 $+\mathrm{T}$ cells were sequenced as 150 bp pairend reads. Sequencing datasets were analyzed to assign reads to the BL6 genome using the NCBI build $\mathrm{v} 38 / \mathrm{mm} 10$ reference genome assembly obtained from the UCSC Genome Browser using Bowtie2. Differential tracks and heatmaps were generated by Deeptools. GO analysis was done using http://geneontology.org/.

\section{Data availability}

All sequencing data that support the findings of this study have been deposited in the National Centre for Biotechnology Information GEO and are accessible through the GEO SuperSeries "GSE59779". Publicly available RNA Immunoprecipitation data for Fig. 2i were obtained from NCBI GEO "GSE118402" (RIP-seq_TSC HNRNPK) and "GSE137491" (WT_1_ESC_SUZ12_RIPseq and WT_1_ESC_EZH2_RIPseq). All other data and the scripts used for the analyses that support the findings of this study are available within the article and its Supplementary Information files or from the corresponding authors upon reasonable request. Source data are provided with this paper. A reporting summary for this Article is available as a Supplementary Information file. Source data are provided with this paper.

Received: 5 July 2019; Accepted: 28 October 2020;

Published online: 27 November 2020

\section{References}

1. Augui, S., Nora, E. P. \& Heard, E. Regulation of X-chromosome inactivation by the X-inactivation centre. Nat. Rev. Genet. 12, 429-442 (2011).

2. Galupa, R. \& Heard, E. X-chromosome inactivation: a crossroads between chromosome architecture and gene regulation. Annu. Rev. Genet. 52, 535-566 (2018).

3. Engreitz, J. M. et al. The Xist lncRNA exploits three-dimensional genome architecture to spread across the X chromosome. Science 341, 1237973 (2013).

4. Simon, M. D. et al. High-resolution Xist binding maps reveal two-step spreading during X-chromosome inactivation. Nature 504, 465-469 (2013).

5. Heard, E. \& Disteche, C. M. Dosage compensation in mammals: fine-tuning the expression of the X chromosome. Genes Dev. 20, 1848-1867 (2006).

6. Mira-Bontenbal, H. \& Gribnau, J. New Xist-interacting proteins in Xchromosome inactivation. Curr. Biol. 26, R338-R342 (2016).

7. Pinheiro, I. \& Heard, E. X chromosome inactivation: new players in the initiation of gene silencing. F1000Res 6 Faculty Rev-344. (2017).

8. Żylicz, J. J. et al. The implication of early chromatin changes in X chromosome inactivation. Cell 176, 182-197.e23 (2019).

9. Bonora, G. \& Disteche, C. M. Structural aspects of the inactive X chromosome. Philos. Trans. R. Soc. Lond. B Biol. Sci. 372, 1733 (2017).

10. Jégu, T., Aeby, E. \& Lee, J. T. The X chromosome in space. Nat. Rev. Genet. 18 377-389 (2017).

11. Andrulis, E. D., Neiman, A. M., Zappulla, D. C. \& Sternglanz, R. Perinuclear localization of chromatin facilitates transcriptional silencing. Nature 394, 592-595 (1998).

12. Barr, M. L. \& Bertram, E. G. A morphological distinction between neurones of the male and female, and the behaviour of the nucleolar satellite during accelerated nucleoprotein synthesis. Nature 163, 676 (1949).

13. Lyon, M. F. Sex chromatin and gene action in the mammalian Xchromosome. Am. J. Hum. Genet. 14, 135-148 (1962).

14. Rego, A., Sinclair, P. B., Tao, W., Kireev, I. \& Belmont, A. S. The facultative heterochromatin of the inactive $\mathrm{X}$ chromosome has a distinctive condensed ultrastructure. J. Cell Sci. 121, 1119-1127 (2008).

15. Zhang, L. F., Huynh, K. D. \& Lee, J. T. Perinucleolar targeting of the inactive X during S phase: evidence for a role in the maintenance of silencing. Cell 129, 693-706 (2007).

16. Padeken, J. \& Heun, P. Nucleolus and nuclear periphery: velcro for heterochromatin. Curr. Opin. Cell Biol. 28, 54-60 (2014).

17. Belagal, P. et al. Decoding the principles underlying the frequency of association with nucleoli for RNA polymerase III-transcribed genes in budding yeast. Mol. Biol. Cell 27, 3164-3177 (2016).

18. Huang, S., Deerinck, T. J., Ellisman, M. H. \& Spector, D. L. The dynamic organization of the perinucleolar compartment in the cell nucleus. J. Cell Biol. 137, 965-974 (1997).

19. Chen, C. K. et al. Xist recruits the X chromosome to the nuclear lamina to enable chromosome-wide silencing. Science 354, 468-472 (2016).

20. Yang, F. et al. The IncRNA Firre anchors the inactive $\mathrm{X}$ chromosome to the nucleolus by binding CTCF and maintains $\mathrm{H} 3 \mathrm{~K} 27 \mathrm{me} 3$ methylation. Genome Biol. 16, 52 (2015).

21. Hacisuleyman, E., Shukla, C. J., Weiner, C. L. \& Rinn, J. L. Function and evolution of local repeats in the Firre locus. Nat. Commun. 7, 11021 (2016).

22. Barutcu, A. R., Maass, P. G., Lewandowski, J. P., Weiner, C. L. \& Rinn, J. L. A TAD boundary is preserved upon deletion of the CTCF-rich Firre locus. Nat. Commun. 9, 1444 (2018).

23. Hacisuleyman, E. et al. Topological organization of multichromosomal regions by the long intergenic noncoding RNA Firre. Nat. Struct. Mol. Biol. 21, 198-206 (2014)

24. Lu, Y. et al. The NF-kB-responsive long noncoding RNA FIRRE regulates posttranscriptional regulation of inflammatory gene expression through interacting with hnRNPU. J. Immunol. 199, 3571-3582 (2017).

25. Izuogu, O. G. et al. Analysis of human ES cell differentiation establishes that the dominant isoforms of the IncRNAs RMST and FIRRE are circular. BMC Genomics 19, 276 (2018).

26. Rao, S. S. et al. A 3D map of the human genome at kilobase resolution reveals principles of chromatin looping. Cell 159, 1665-1680 (2014).

27. Darrow, E. M. et al. Deletion of DXZ4 on the human inactive X chromosome alters higher-order genome architecture. Proc. Natl Acad. Sci. USA 113, E4504-E4512 (2016).

28. Horakova, A. H., Moseley, S. C., McLaughlin, C. R., Tremblay, D. C. \& Chadwick, B. P. The macrosatellite DXZ4 mediates CTCF-dependent longrange intrachromosomal interactions on the human inactive $\mathrm{X}$ chromosome. Hum. Mol. Genet. 21, 4367-4377 (2012).

29. Bonora, G. et al. Orientation-dependent Dxz4 contacts shape the 3D structure of the inactive X chromosome. Nat. Commun. 9, 1445 (2018).

30. Deng, X. et al. Bipartite structure of the inactive mouse X chromosome. Genome Biol. 16, 152 (2015).

31. Giorgetti, L. et al. Structural organization of the inactive $\mathrm{X}$ chromosome in the mouse. Nature 535, 575-579 (2016). 
32. Andergassen, D. et al. In vivo Firre and Dxz4 deletion elucidates roles for autosomal gene regulation. elife 8, e47214 (2019).

33. Lewandowski, J. P. et al. The Firre locus produces a trans-acting RNA molecule that functions in hematopoiesis. Nat. Commun. 10, 5137 (2019).

34. Andergassen, D. et al. Mapping the mouse Allelome reveals tissue-specific regulation of allelic expression. elife 6, e25125 (2017).

35. Froberg, J. E., Pinter, S. F., Kriz, A. J., Jégu, T. \& Lee, J. T. Megadomains and superloops form dynamically but are dispensable for X-chromosome inactivation and gene escape. Nat. Commun. 9, 5004 (2018).

36. Luense, S. et al. Quantification of histone $\mathrm{H} 3$ Lys27 trimethylation (H3K27me3) by high-throughput microscopy enables cellular large-scale screening for small-molecule EZH2 inhibitors. J. Biomol. Screen 20, 190-201 (2015).

37. Marks, $\mathrm{H}$. et al. High-resolution analysis of epigenetic changes associated with X inactivation. Genome Res. 19, 1361-1373 (2009).

38. Thakur, J., He, F., Trizia, L., Christine, D. \& Steven, H. Architectural RNA is required for heterochromatin organization. biorvix https://doi.org/10.1101/ 784835 (2019).

39. Schertzer, M. D. et al. IncRNA-induced spread of polycomb controlled by genome architecture, RNA abundance, and CpG Island DNA. Mol. Cell 75, 523-537.e10 (2019).

40. Garland, W. et al. A functional link between nuclear RNA decay and transcriptional control mediated by the polycomb repressive complex 2. Cell Rep. 29, 1800-1811.e6 (2019).

41. Wei, C. et al. RBFox2 binds nascent RNA to globally regulate polycomb complex 2 targeting in mammalian genomes. Mol. Cell 62, 875-889 (2016).

42. Kaneko, S. et al. Interactions between JARID2 and noncoding RNAs regulate PRC2 recruitment to chromatin. Mol. Cell 53, 290-300 (2014).

43. Kaneko, S., Son, J., Shen, S. S., Reinberg, D. \& Bonasio, R. PRC2 binds active promoters and contacts nascent RNAs in embryonic stem cells. Nat. Struct. Mol. Biol. 20, 1258-1264 (2013).

44. Tavares, L. et al. RYBP-PRC1 complexes mediate H2A ubiquitylation at polycomb target sites independently of PRC2 and H3K27me3. Cell 148, 664-678 (2012).

45. Rosenberg, M. et al. Denaturing CLIP, dCLIP, pipeline identifies discrete RNA footprints on chromatin-associated proteins and reveals that CBX7 targets 3' UTRs to regulate mRNA expression. Cell Syst. 5, 368-385.e15 (2017).

46. Huang, Y. H. et al. 2B4-SAP signaling is required for the priming of naive CD8. Oncoimmunology 6, el267094 (2017).

47. Yamada, T., Park, C. S., Mamonkin, M. \& Lacorazza, H. D. Transcription factor ELF4 controls the proliferation and homing of CD8 $+\mathrm{T}$ cells via the Krüppel-like factors KLF4 and KLF2. Nat. Immunol. 10, 618-626 (2009).

48. Ashburner, M. et al. Gene ontology: tool for the unification of biology. The Gene Ontology Consortium. Nat. Genet. 25, 25-29 (2000).

49. Huang, N., Lee, I., Marcotte, E. M. \& Hurles, M. E. Characterising and predicting haploinsufficiency in the human genome. PLoS Genet. 6, e1001154 (2010).

50. Gao, F., Cai, Y., Kapranov, P. \& Xu, D. Reverse-genetics studies of lncRNAswhat we have learnt and paths forward. Genome Biol. 21, 93 (2020).

51. Yusufzai, T. M., Tagami, H., Nakatani, Y. \& Felsenfeld, G. CTCF tethers an insulator to subnuclear sites, suggesting shared insulator mechanisms across species. Mol. Cell 13, 291-298 (2004).

52. Chen, L. L. Linking long noncoding RNA localization and function. Trends Biochem. Sci. 41, 761-772 (2016)

53. Fatica, A. \& Bozzoni, I. Long non-coding RNAs: new players in cell differentiation and development. Nat. Rev. Genet. 15, 7-21 (2014).

54. Vance, K. W. \& Ponting, C. P. Transcriptional regulatory functions of nuclear long noncoding RNAs. Trends Genet. 30, 348-355 (2014).

55. Engreitz, J. M. et al. Local regulation of gene expression by lncRNA promoters, transcription and splicing. Nature 539, 452-455 (2016).

56. Engreitz, J. M., Ollikainen, N. \& Guttman, M. Long non-coding RNAs: spatial amplifiers that control nuclear structure and gene expression. Nat. Rev. Mol. Cell Biol. 17, 756-770 (2016).

57. Zhao, J., Sun, B. K., Erwin, J. A., Song, J. J. \& Lee, J. T. Polycomb proteins targeted by a short repeat RNA to the mouse X chromosome. Science 322, 750-756 (2008).

58. Kanhere, A. et al. Short RNAs are transcribed from repressed polycomb target genes and interact with polycomb repressive complex-2. Mol. Cell 38, 675-688 (2010).

59. da Rocha, S. T. et al. Jarid2 is implicated in the initial Xist-induced targeting of PRC2 to the inactive X chromosome. Mol. Cell 53, 301-316 (2014).

60. Pintacuda, G. et al. hnRNPK recruits PCGF3/5-PRC1 to the Xist RNA Brepeat to establish polycomb-mediated chromosomal silencing. Mol. Cell 68, 955-969.e10 (2017).

61. Plath, K. et al. Role of histone $\mathrm{H} 3$ lysine 27 methylation in $\mathrm{X}$ inactivation. Science 300, 131-135 (2003).

62. Graindorge, A. et al. In-cell identification and measurement of RNA-protein interactions. Nat. Commun. 10, 5317 (2019).
63. Brockdorff, N. Polycomb complexes in X chromosome inactivation. Philos. Trans. R. Soc. Lond. B Biol. Sci. 372, 20170021 (2017).

64. Lu, Z., Carter, A. C. \& Chang, H. Y. Mechanistic insights in X-chromosome inactivation. Philos. Trans. R. Soc. Lond. B Biol. Sci. 372, 20160356 (2017).

65. Brockdorff, N., Bowness, J. S. \& Wei, G. Progress toward understanding chromosome silencing by Xist RNA. Genes Dev. 34, 733-744 (2020).

66. Monfort, A. \& Wutz, A. The B-side of Xist. F1000Res 9 F1000 Faculty Rev-55. (2020).

67. Youmans, D. T., Schmidt, J. C. \& Cech, T. R. Live-cell imaging reveals the dynamics of PRC2 and recruitment to chromatin by SUZ12-associated subunits. Genes Dev. 32, 794-805 (2018).

68. Grote, P. et al. The tissue-specific lncRNA Fendrr is an essential regulator of heart and body wall development in the mouse. Dev. Cell 24, 206-214 (2013).

69. Marín-Béjar, O. et al. Pint lincRNA connects the p53 pathway with epigenetic silencing by the Polycomb repressive complex 2. Genome Biol. 14, R104 (2013).

70. Yen, Y. P. et al. locus-derived lncRNAs perpetuate postmitotic motor neuron cell fate and subtype identity. elife 7, e38080 (2018).

71. Das, P. P. et al. PRC2 is required to maintain expression of the maternal Gt12Rian-Mirg locus by preventing de novo DNA methylation in mouse embryonic stem cells. Cell Rep. 12, 1456-1470 (2015).

72. Zhao, J. et al. Genome-wide identification of polycomb-associated RNAs by RIP-seq. Mol. Cell 40, 939-953 (2010).

73. Padeken, J. et al. The nucleoplasmin homolog NLP mediates centromere clustering and anchoring to the nucleolus. Mol. Cell 50, 236-249 (2013).

74. Holmberg Olausson, K., Nistér, M. \& Lindström, M. S. Loss of nucleolar histone chaperone NPM1 triggers rearrangement of heterochromatin and synergizes with a deficiency in DNA methyltransferase DNMT3A to drive ribosomal DNA transcription. J. Biol. Chem. 289, 34601-34619 (2014).

75. Lyu, G. et al. Changes in the position and volume of inactive $\mathrm{X}$ chromosomes during the G0/G1 transition. Chromosome Res. 26, 179-189 (2018).

76. Stewart, E. R. et al. Maintenance of epigenetic landscape requires CIZ1 and is corrupted in differentiated fibroblasts in long-term culture. Nat. Commun. 10, 460 (2019).

77. Colognori, D., Sunwoo, H., Kriz, A. J., Wang, C. Y. \& Lee, J. T. Xist deletional analysis reveals an interdependency between Xist RNA and polycomb complexes for spreading along the inactive X. Mol. Cell 74, 101-117.e10 (2019).

78. Saldaña-Meyer, R. et al. RNA interactions are essential for CTCF-mediated genome organization. Mol. Cell 76, 412-422.e5 (2019).

79. Ezhkova, E. et al. EZH1 and EZH2 cogovern histone H3K27 trimethylation and are essential for hair follicle homeostasis and wound repair. Genes Dev. 25, 485-498 (2011).

80. Kim, J. M. et al. Linker histone H1.2 establishes chromatin compaction and gene silencing through recognition of H3K27me3. Sci. Rep. 5, 16714 (2015).

81. Hasegawa, Y., Brockdorff, N., Kawano, S., Tsutui, K. \& Nakagawa, S. The matrix protein hnRNP $\mathrm{U}$ is required for chromosomal localization of Xist RNA. Dev. Cell 19, 469-476 (2010).

82. Pullirsch, D. et al. The Trithorax group protein Ash2l and Saf-A are recruited to the inactive $\mathrm{X}$ chromosome at the onset of stable $\mathrm{X}$ inactivation. Development 137, 935-943 (2010).

83. McHugh, C. A. et al. The Xist lncRNA interacts directly with SHARP to silence transcription through HDAC3. Nature 521, 232-236 (2015).

84. Fan, H. et al. The nuclear matrix protein HNRNPU maintains 3D genome architecture globally in mouse hepatocytes. Genome Res. 28, 192-202 (2018).

85. Goff, L. A. \& Rinn, J. L. Linking RNA biology to lncRNAs. Genome Res. 25, 1456-1465 (2015).

86. Beltrán-Anaya, F. O., Cedro-Tanda, A., Hidalgo-Miranda, A. \& RomeroCordoba, S. L. Insights into the regulatory role of non-coding RNAs in cancer metabolism. Front. Physiol. 7, 342 (2016).

87. Nóbrega, M. A., Zhu, Y., Plajzer-Frick, I., Afzal, V. \& Rubin, E. M. Megabase deletions of gene deserts result in viable mice. Nature 431, 988-993 (2004).

88. Han, X. et al. Mouse knockout models reveal largely dispensable but contextdependent functions of IncRNAs during development. J. Mol. Cell Biol. 10, 175-178 (2018).

89. Rossi, A. et al. Genetic compensation induced by deleterious mutations but not gene knockdowns. Nature 524, 230-233 (2015).

90. Son, J., Shen, S. S., Margueron, R. \& Reinberg, D. Nucleosome-binding activities within JARID2 and EZH1 regulate the function of PRC2 on chromatin. Genes Dev. 27, 2663-2677 (2013).

91. Fang, J. et al. PIRCh-seq: functional classification of non-coding RNAs associated with distinct histone modifications. Genome Biol. 20, 292 (2019).

92. Wang, J. et al. Unusual maintenance of $\mathrm{X}$ chromosome inactivation predisposes female lymphocytes for increased expression from the inactive $\mathrm{X}$. Proc. Natl Acad. Sci. USA 113, E2029-E2038 (2016).

93. Lingenfelter, P. A. et al. Escape from X inactivation of Smcx is preceded by silencing during mouse development. Nat. Genet. 18, 212-213 (1998). 
94. Yang, F., Babak, T., Shendure, J. \& Disteche, C. M. Global survey of escape from $\mathrm{X}$ inactivation by RNA-sequencing in mouse. Genome Res. 20, 614-622 (2010).

95. Berletch, J. B. et al. Escape from $\mathrm{X}$ inactivation varies in mouse tissues. PLoS Genet. 11, e1005079 (2015).

96. Skene, P. J., Henikoff, J. G. \& Henikoff, S. Targeted in situ genome-wide profiling with high efficiency for low cell numbers. Nat. Protoc. 13, 1006-1019 (2018).

\section{Acknowledgements}

This work was supported by grants GM131745 (CMD) and GM127327 (XD) from the National Institute of General Medical Sciences, by grant DK107979 (JS and WSN) from the National Institutes of Health Common Fund 4D Nucleome, and by a HHMI Faculty Scholars grant (J.L.R.). We thank the HSCRB Flow Cytometry Core for assistance with cell sorting, the Bauer Sequencing Core at Harvard University for sequencing, and Nydia Chang for assistance with mouse husbandry.

\section{Author contributions}

H.F., G.B., X.D., W.S.N., and C.M.D. conceived the study; H.F. and X.D. constructed the mutant cell lines; J.P.L. and J.L.R. constructed the mouse model; J.T. and S.H. performed CUT\&RUN; H.F., G.N.F., Z.D., and J.S. performed RNA-seq, ATAC-seq, ChIP-seq, and Hi-C assays; G.B. and H.F. performed data analyses; H.F., G.B., X.D., W.S.N., and C.M.D. wrote the manuscript, with input from all authors.

\section{Competing interests}

The authors declare no competing interests.

\section{Additional information}

Supplementary information is available for this paper at https://doi.org/10.1038/s41467020-19879-3.

Correspondence and requests for materials should be addressed to X.D., W.S.N. or C.M.D.

Peer review information Nature Communications thanks the anonymous reviewer(s) for their contribution to the peer review of this work.

Reprints and permission information is available at http://www.nature.com/reprints

Publisher's note Springer Nature remains neutral with regard to jurisdictional claims in published maps and institutional affiliations.

(c) (i) Open Access This article is licensed under a Creative Commons Attribution 4.0 International License, which permits use, sharing, adaptation, distribution and reproduction in any medium or format, as long as you give appropriate credit to the original author(s) and the source, provide a link to the Creative Commons license, and indicate if changes were made. The images or other third party material in this article are included in the article's Creative Commons license, unless indicated otherwise in a credit line to the material. If material is not included in the article's Creative Commons license and your intended use is not permitted by statutory regulation or exceeds the permitted use, you will need to obtain permission directly from the copyright holder. To view a copy of this license, visit http://creativecommons.org/ licenses/by/4.0/.

(C) The Author(s) 2020 